See discussions, stats, and author profiles for this publication at: https://www.researchgate.net/publication/324969117

\title{
Multi-Output Tree Chaining: An Interpretative Modelling and Lightweight Multi-Target Approach
}

Article in Journal of Signal Processing Systems · May 2018

Dol: 10.1007/s11265-018-1376-5

\section{CITATIONS}

10

7 authors, including:

Saulo Martiello Mastelini

University of São Paulo

35 PUBLICATIONS 122 CITATIONS

SEE PROFILE

Everton Santana

Universidade Estadual de Londrina

15 PUBLICATIONS 59 CITATIONS

SEE PROFILE
READS

75

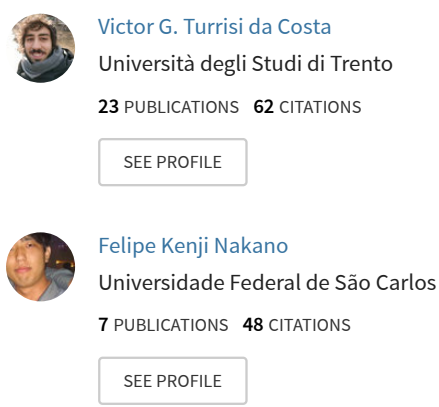

Some of the authors of this publication are also working on these related projects:

Project Mobile botnet detection View project

Project Machine Learning characterization of food quality datasets View project 


\title{
Multi-Output Tree Chaining: An Interpretative Modelling and Lightweight Multi-Target Approach
}

\author{
Saulo Martiello Mastelini ${ }^{1}$ (D) Victor Guilherme Turrisi da Costa ${ }^{1} \cdot$ Everton Jose Santana $^{2} \cdot$ Felipe Kenji Nakano $^{3}$. \\ Rodrigo Capobianco Guido ${ }^{4}$. Ricardo Cerri ${ }^{3}$. Sylvio Barbon Jr. ${ }^{1}$
}

Received: 15 September 2017 / Revised: 16 January 2018 / Accepted: 27 April 2018

(C) Springer Science+Business Media, LLC, part of Springer Nature 2018

\begin{abstract}
Multi-target regression (MTR) regards predictive problems with multiple numerical targets. To solve this, machine learning techniques can model solutions treating each target as a separated problem based only on the input features. Nonetheless, modelling inter-target correlation can improve predictive performance. When performing MTR tasks using the statistical dependencies of targets, several approaches put aside the evaluation of each pair-wise correlation between those targets, which may differ for each problem. Besides that, one of the main drawbacks of the current leading MTR method is its high memory cost. In this paper, we propose a novel MTR method called Multi-output Tree Chaining (MOTC) to overcome the mentioned disadvantages. Our method provides an interpretative internal tree-based structure which represents the relationships between targets denominated Chaining Trees (CT). Different from the current techniques, we compute the outputs dependencies, one-by-one, based on the Random Forest importance metric. Furthermore, we proposed a memory friendly approach which reduces the number of required regression models when compared to a leading method, reducing computational cost. We compared the proposed algorithm against three MTR methods (Single-target - ST; Multi-Target Regressor Stacking - MTRS; and Ensemble of Regressor Chains - ERC) on 18 benchmark datasets with two base regression algorithms (Random Forest and Support Vector Regression). The obtained results show that our method is superior to the ST approach regarding predictive performance, whereas, having no significant difference from ERC and MTRS. Moreover, the interpretative tree-based structures built by MOTC pose as great insight on the relationships among targets. Lastly, the proposed solution used significantly less memory than ERC being very similar in predictive performance.
\end{abstract}

Keywords Multi-target regression $\cdot$ Multi-output $\cdot$ Memory-friendly algorithm $\cdot$ Interpretative tree structure $\cdot$ Machine learning

Saulo Martiello Mastelini mastelini@uel.br

Victor Guilherme Turrisi da Costa

victorturrisi@uel.br

Everton Jose Santana

santana.everton@ieee.org

Felipe Kenji Nakano

felipe.nakano@dc.ufscar.br

Rodrigo Capobianco Guido

guido@ieee.org

Ricardo Cerri

cerri@dc.ufscar.br

Sylvio Barbon Jr.

barbon@uel.br

1 Computer Science Department, State University of Londrina. Rodovia Celso Garcia Cid, Km 380, s/n - Campus Universitário, Londrina, PR, 86057-970, Brazil

\section{Introduction}

The concept of machine learning (ML) denotes a wide set of techniques that have been explored in multiple fields with a diverse set of objectives. One of those research fields is the signal processing, which consists of several tasks, such as discovering important features from noisy signals,

2 Electrical Engineering Department, State University of Londrina. Rodovia Celso Garcia Cid, Km 380, s/n - Campus Universitário, Londrina, PR, 86057-970, Brazil

3 Department of Computer Science, Federal University of São Carlos, Rodovia Washington Luís, km 235, São Carlos, SP 13565-905, Brazil

4 Instituto de Biociências, Letras e Ciências Exatas, Unesp - Univ Estadual Paulista (São Paulo State University), Rua Cristóvão Colombo 2265, Jd Nazareth, 15054-000, São José do Rio Preto, SP, Brazil 
enhancing signal quality and predicting signals properties $[4,5,8,10,14,18,24]$. One frequently performed task consists of predicting a target, response, or output, $y$ based on a set $X_{m}$ of $m$ input variables. These target values can be categorical or numerical, resulting in classification or regression problems, respectively. Categorical targets assume a finite set of $L$ possible output values. When $|L|=$ 2 , the resulting task is denominated a binary predictive problem. Whenever $|L|>2$, the obtained problem is called a multiclass classification task. In contrast, when regression tasks are considered, the output variable assumes ordered and infinite numerical values.

When a learning task focus on predicting a unique target, we have a single-target (ST) problem. On the other hand, many machine learning problems consist of multiple targets, which are denominated as multi-target (MT) problems [2, 17]. MT includes many types of prediction tasks depending on the type of the responses, i.e., multi-label, multidimensional, hierarchical classification, and multi-target regression $[2,17,21]$.

Consider a description space $X$ that consists of $i$ tuples with primitive data types (discrete or continuous values) in the form $X_{i}=\left\{x_{i_{1}}, x_{i_{2}}, \ldots, x_{i_{m}}\right\}$, where $m$ represents the number of descriptive features. Also, consider a target space $Y$ in the form $Y_{i}=\left\{y_{i_{1}}, y_{i_{2}}, \ldots, y_{i_{d}}\right\}$, being $d$ the number of problem's outputs. In addition, take a set of examples $E$ comprising elements from $X$ and $Y$, i.e., $E=\left\{\left(X_{i}, Y_{i}\right)\right\}$, $1 \leq i \leq N$, where $N$ is the number of examples in $E$. Lastly, take into consideration a quality criterion $q$ which rewards prediction models with high predictive performance and low complexity [17]. A MT problem consists in finding a function $f: X \rightarrow Y$ such that $f$ maximises $q$. The mentioned prediction problem is solved upon the set of know examples, $E$. In a MT problem, $f$ can either refer to a single prediction model or a set thereof.

Depending on $Y$, different denominations are given to the modelling. When the elements in $Y$ assume binary values, the resulting task is called Multi-label classification [17], in contrast to multi-dimensional or multi-target classification, which is related to the prediction of multiple discrete or categorical values that assume more than two single values $[17,21]$. In hierarchical classification tasks, the multiple outputs are arranged into a directed acyclic graph or in a tree hierarchy [17]. When the elements of $Y$ are continuous, with the possibility of being statistically correlated, the related predictive problem is called multi-target regression (MTR) $[1,2,21,25,26]$.

In some cases, one could simply consider that the targets have no underlying relational properties and treat the prediction of each target as a separated predictive task, applying a ST technique. This comprehends to the extensive set of solutions based on machine learning, e.g., Artificial Neural Networks (ANN), Decision Trees (DT),
Bayesian Models and Support Vector Machines (SVM). However, this assumption causes a negative impact on prediction performance since the inter-correlation between targets are ignored. In fact, many real-life problems such as the prediction of multiple vegetation, soil, water and river flow properties, the estimation of monthly online product sales, the price forecasting of multiple products in a supply management chain and of air-tickets for different companies, and even solar flares [2, 15, 25], are reported as MTR tasks. Whenever targets have dependencies among themselves, the easier to predict can be used as additional features for the more challenging ones. Nevertheless, the ST strategy has been employed across multiple MTR papers as a base level method for comparisons [2, 21, 25].

In the context of ML for signal processing, many works have been published in the past years $[4,5,8,24,27,28]$. In these works, regression models have been broadly applied in time series, text, speech and image processing, for instance in the determination of sound sources direction-of-arrival, estimation of the head pose, improvement of low-resolution images and prediction of stock prices. In these kind of tasks, the computational costs of a solution can limit its application even if it has high predictive capabilities.

According to Borchani et al. [2], MTR tasks are solved with two general approaches: algorithm adaptation and problem transformation. Kocev et al. [17] denote the methodologies as global and local methods, respectively. The former refers to the adaptation of well known ST regression algorithms to deal with multiple outputs while additionally investigating the possible relationships among targets. In the latter, the training data is manipulated, and common regression techniques to predict ST problems are then employed. Several MTR methods belonging to both approaches were proposed in past years $[1,2,17,21,22$, 25]. Some of the local approaches had achieved the highest performance in multiple MT scenarios, outperforming global methods [21, 22, 25, 26]. However, the actual state-of-art methods present some limitations in the interpretation of targets dependencies and have high computational cost solutions.

In this paper, we propose a novel local method, called Multi-output Tree Chaining (MOTC), which uses a treebased representation to model the statistical dependencies between targets. Our goal is to address the aforementioned disadvantages by creating a highly interpretable with high predictive performance method, whereas being memory and time conservative when constructing the chaining trees. MOTC differs from other local-based methods in the sense that it uses only the existing dependency relationships between outputs as additional information for regression, with a sophisticated and yet lightweight approach. Also, we propose to use the Random Forest variable importance metric to model nonlinear statistical dependencies between targets. Using the Hoeffding Bound concept [13], we select 
groups of outputs which are the most correlated with a specific response, without memory-time trade-offs.

Through an extensible experimental evaluation of MOTC against three other local-based MTR methods (Singletarget, Multi-target Regressor Stacking and Ensemble of Regressor Chains) in 18 benchmark datasets along with two regression techniques (Random Forest and Support Vector Machine), we demonstrate that our method can achieve high prediction performance, whereas generating a compact number of regression models when compared with ERC, reducing the memory cost. Besides, we present a combined representation of the generated MOTC's chaining trees to give some insights about how they could be employed as an interpretation tool. In this way, it is possible to discover linear and nonlinear target relations and contribute toward further advances in signal processing technologies.

The rest of this paper is structured as follows. Section 2 reviews related works in MTR. In Section 3, our method (MOTC) is presented. Section 4 presents the materials and the experimental setup employed in our experiments. In Section 5 we discuss the obtained results, comparing MOTC with other MTR approaches. We present the final considerations in Section 6. Lastly, two Appendices with the description of the datasets used in the work and the condensed representations of the chaining trees obtained during the experiments are included.

\section{Related Works}

A first directly derived approach of problem transformation is to predict each target variable separately, as multiple independent ST problems. Although pretty straightforward, this method outperforms MTR methods (both those based on algorithm adaptation and problem transformation) in many cases [2, 25]. Nonetheless, ST approaches do not explore the assumed target correlations, so using an MTR strategy ought to achieve better results when there are target co-dependencies. Although more than one predictor is used to represent an MTR problem, which leads to negatively impacting the generated model interpretation and increasing computation training costs, this kind of approach offers multiple advantages. Firstly, the possibility of using any base regressor, or even a hybrid set of regressors, could lead to better prediction performance and exploration of a particular problem characteristics. Besides that, problem adaptation methods improve the resulting model modularity and conceptual simplicity, presenting better accuracy than state-of-the-art methods [21, 22, 25].

In past years, some problem transformation methods were proposed to exploit inter-target properties as well. Zhang et al. [29] proposed the modification of the input space of a task through a visualisation technique so that a MTR task could be modelled as a wider ST problem. The authors used a Support Vector Regression (SVR) machine and achieved results comparable to the ST strategy. Tsoumakas et al. [26] created random linear combinations of the targets to explore the relation between output values. This approach increases the original feature space dimension and solves multiple ST problems in the transformed space. After that, the values predicted are used to solve a linear system to obtain the original target predictions. Their results compared favourably both for the ST approach and the global-based solution Multi-objective Random Forest (MORF), firstly described in Kocev et al. [16].

Some MTR methods were also adapted from multilabel classification (MLC) [2, 25]. Spyromitros-Xioufis et al. [25] proposed two main methods inspired by MLC: Multi-Target Regressor Stacking (MTRS) and Ensemble of Regressor Chains (ERC). Both have the core concept of applying targets approximations as additional, or expanded, features, as proposed in the Cascade Generalisation [11]. These methods are better described in Sections 2.1 and 2.2.

A few recent works were inspired by the idea of the MTRS, with the premise that deeper layers of stacked ST predictors could offer better predictive performance than using just one layer (ST) or two layers (MTRS). Santana et al. [23] proposed the Deep Regressor Stack (DRS), which uses the targets estimations as additional predicting features in a naive deep learning method. In DRS, the predictions of the targets that obtained less error on a validation dataset are sequentially added as new features, with a fixed number of layers. Their approach presented some gains over the other compared MTR solutions, though this method brought an overhead both in time and memory.

Another promising novel approach, called Deep Structure for Tracking Asynchronous Regressor Stacking (DSTARS) was proposed by Mastelini et al. [20]. By defining the best number of regression layers in an adaptive manner, DSTARS is able to model different levels of interaction between targets and could bring some clues about their relationships. DSTARS has the drawback of increasing computation memory and time cost due to the use of deeper layers of stacked ST predictors, as well as, a processing stage to find the adequate adequate number of layers built for each output.

Aiming at improving the ERC algorithm, two localbased MTR methods were proposed recently. Moyano et al. [22] proposed the use of Genetic Algorithm (GA) to find an improved set of target chains and to explore the linear correlation among target variables. Their method performs better than ERC in some scenarios, but solving the combinatory problem brings a new processing overhead to the already very costly ERC algorithm.

Melki et al. [21] proposed the Maximum Correlated Chain method with a single group of chained ST regressors 
instead of an ensemble of chain predictors. They computed the linear correlation between each pair of target variables to determine the order in which models would be induced. Each model, in the same way as other local-based approaches, uses the preceding regressors predictions as additional features. The authors report good prediction results when comparing their approach with ERC, while also converting the set of chains to a single group, reducing computational time and memory complexities.

Both ERC-based approaches use the linear correlation to explain the underlying relationships among target variables. Despite the assumption of linear dependency is enough in many problems, some tasks are subject to present non-linear statistical relationships between targets. In those tasks, non-linear behaviour mapping should reflect in better problem comprehension and greater predictive performance. Besides that, the aforementioned works assume that the maximisation of the summed correlation within chains is a complete descriptor of the inter-responses dependency behaviour. They do not consider different degrees of correlation between them, as each target is preceded only by a unique assumed less correlated response, in a queue-like fashion.

Our approach overcomes the mentioned gaps in ERCbased approaches. By employing the Random Forest (RF) importance metric, MOTC is able to quantify non-linear relations among target variables. Also, by representing the dependencies among outputs in a tree based representation, multiple levels of dependency are explored. Our tree chaining idea combines both ERC's chains with MTRS's stacked predictors. A path from root node to a leaf could be interpreted as a regression chain, where each level in the chaining tree is equivalent to a MTRS model. MOTC is still able to reduce computational complexities by employing the statistical measure called Hoeffding bound over the measured inter-target dependencies. With this procedure, only the most significant outputs are selected to recursively compose the chaining tree. Lastly, our approach results in highly interpretative models, which enables the study of how targets influence each other in problem-specific domains.

Before presenting the MOTC algorithm, the MTRS and ERC are explained in detail in the following sections, since they provided the conceptual basis to many problem transformation methods, ours included. Besides that, both algorithms are also used in our experimental comparisons against MOTC.

\subsection{Multi-target Regressor Stacking}

MTRS approach concerns additional input features from ST models predictions. In this sense, considering a dataset composed by $X=\left\{x_{1}, x_{2}, \ldots, x_{m}\right\}$ input features and $Y=\left\{y_{1}, y_{2}, \ldots, y_{d}\right\}$ target variables, MTRS adds the $Y^{\prime}=\left\{y_{1}^{\prime}, y_{2}^{\prime}, \ldots, y_{m}^{\prime}\right\}$ ST predictions as new inputs, creating an augmented training dataset $X^{\prime}=$ $\left\{x_{1}, x_{2}, \ldots, x_{m}, y_{1}^{\prime}, y_{2}^{\prime}, \ldots, y_{d}^{\prime}\right\}$. The new dataset is used by each target to train another ST predictors layer, whose outputs are the final predictions of MTRS.

New instances are initially subjected to the first layer of predictors to obtain the output approximations. These outcomes are then merged with the original features which are applied towards the second level of predictors. Therefore, MTRS introduces inter-target relationships by stacking ST models to enhance tasks description and increase prediction performance. However, MTRS does not foresee the modelling of different levels of statistical relationships between outputs, neither does it considers the possible absence of dependencies between them. Also, models generated by the MTRS do not provide insightful information about inter-target properties. The MTRS training procedure is presented in Algorithm 1, where $X$ and $Y$ represent the input and output variables and $d$ the number of targets.

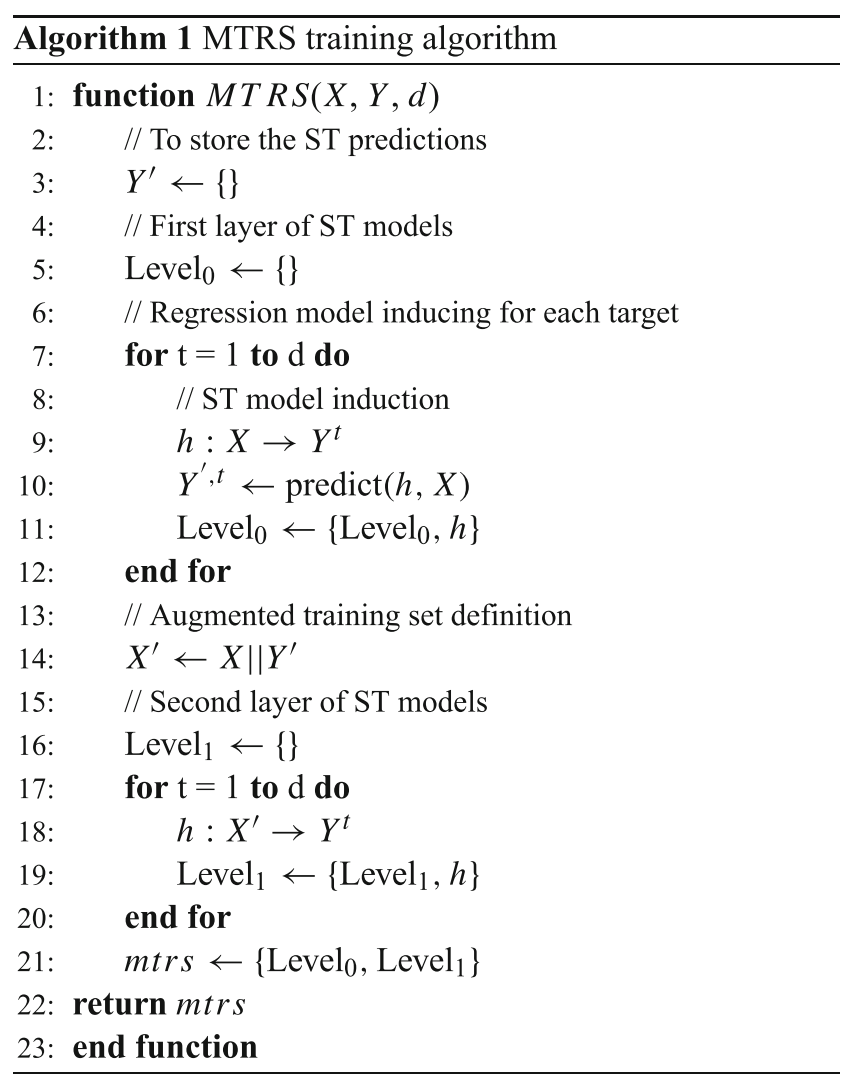

\subsection{Ensemble of Regressor Chains}

The idea behind ERC is to build a set of randomly generated chained ST regressors for each target. Initially, for each chain, a ST model is induced using the first output of the sequence. New models are then induced by 
following the chain order. Each new regressor is trained over the augmented input dataset composed by original input features and the predictions from the preceding regressors in the chain. After training all models, the predicted value of a new instance is the average value obtained from the chain regressors. In other words, the prediction of ERC, for a target $y_{i}, i=[1, d]$, is the average of the $y_{i}$ predicted values over all chains.

Since the output predictions are composed of values from different sorted chains, multiple levels of combinations and inter-dependence between targets are explored. ERC creates all possible target permutations if their number is less than ten $(d \leq 3)$, otherwise, the authors suggest using the fixed value of ten random combinations. The random nature of chain creation does not take into consideration possible relations between targets if they exist. In addition, there is no clear way of interpreting the interactions of responses expressed by a ERC trained model.

The training step of ERC is presented in Algorithm 2. The permute procedure is a function that receives a set of elements and returns all possible permutations among them. It is possible to perform all possible permutations without a limiting parameter, or to specify the maximum number of combinations as an argument (in the original formulation of ERC, the maximum is ten). In the algorithm, $X$ and $Y$ represent the input and output variables and $d$ is the number of targets.

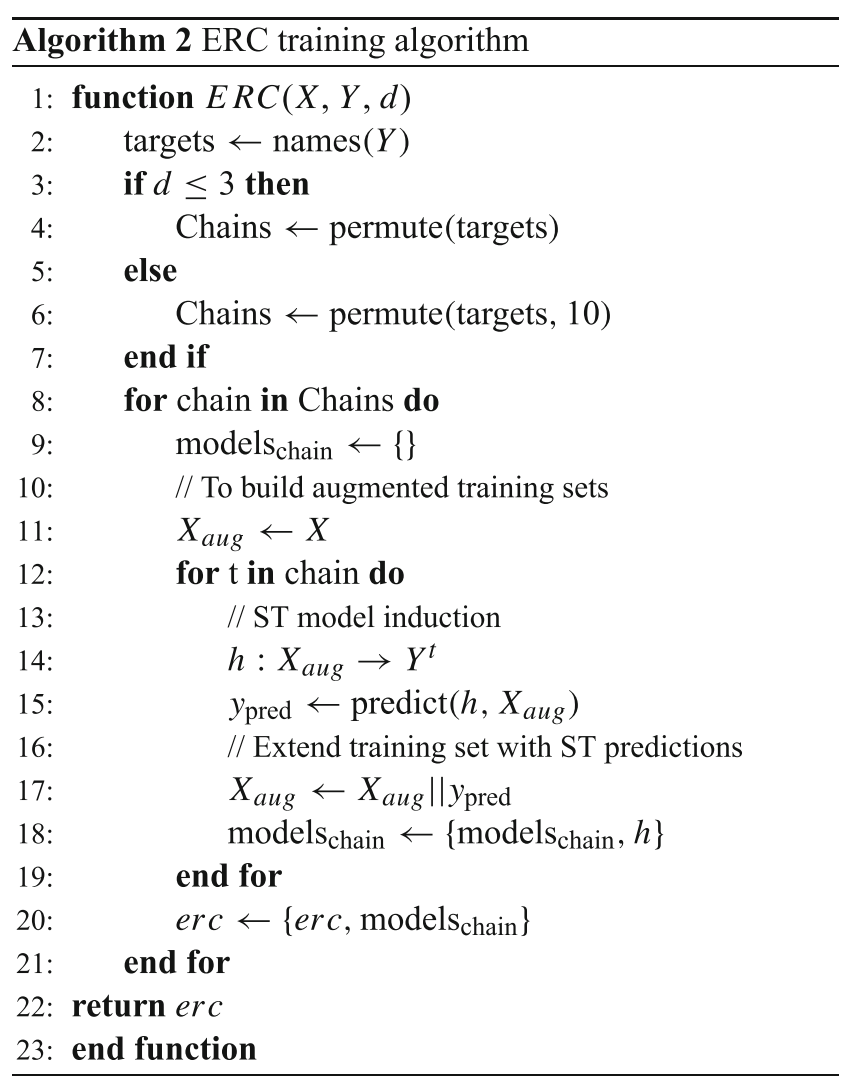

\section{Multi-output Tree Chaining}

As discussed previously, ERC explores different combinations, and thus, various hypotheses of inter-dependencies between targets. This is achieved by randomly ordering the outputs and building a set of chained models. This strategy, besides being time and memory costly, does not sufficiently explore the target combinations and is even subjected to assume non-existent relationships among the outcomes. The method proposed here (MOTC) was projected aiming at both reducing memory consumption and improving the target dependency representation.

In order to truly model different levels of statistical dependencies between target variables, we propose the use of structures in the form of trees to guide the regression models induction. The resulting structures, called Chaining Trees (CTs), are constructed by sequentially evaluating which targets are more correlated with the response being evaluated using the $R F_{\text {imp }}$. MOTC is divided into two steps: a top-down CT construction and a bottom-up regression model induction. In the top-down step, for each target, a CT is built by considering how the outputs are related to each other. Each node in a CT corresponds to a regression model, which is trained during the bottom-up step.

Assume an MTR problem with $X$ input variables and four targets, namely $\mathbf{A}, \mathbf{B}, \mathbf{C}$ and $\mathbf{D}$. Figure 1 shows a $\mathbf{C T}$ for this example when considering $\mathbf{A}$ as the desired output. It is possible to see $\mathbf{A}$, at Level 0 , as the root node and also the targeted variable. Targets $\mathbf{B}$ and $\mathbf{C}$, in this example, represent the outputs which are most correlated to $\mathbf{A}$. Additionally, $\mathbf{B}$ can be better explained using $\mathbf{A}$ and D. This process continues until a maximum CT's depth state is reached, in this case, a maximum level equal to two. It is important to highlight that $\mathbf{A}$ appears both as leaf and root node, stopping the iteration to avoid unnecessary modelling, once $\mathbf{A}$ had already been treated. Chaining trees are constructed in a top-down approach, i.e., from root to leaves, as indicated in Fig. 1.

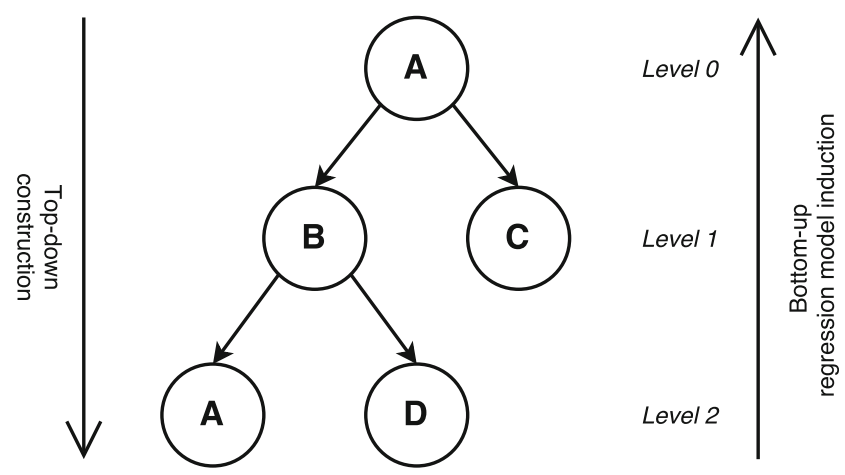

Figure 1 An hypothetical simple example of CT. 
After creating the CT, MOTC performs its final modelling. It consists of inducing ST models in a bottomup fashion, i.e., from leaves to the root node, as presented in Fig. 1. The leaf nodes in the CT correspond to standard ST models, which use only $X$ to describe the desired output. In this sense, ST models are induced for all leaf nodes (A, at Level 2, D and C). After that, MOTC induces a model for $\mathbf{B}$ using an augmented training set which is created by merging $X$ with the predictions of $\mathbf{A}$ and $\mathbf{D}$ done by the previously trained models. Then, at Level $0, \mathbf{A}$ is induced by using as input $X$ and the approximations of $\mathbf{B}$ and $\mathbf{C}$ (both at Level $1)$.

It is clear that a metric should be employed when describing dependencies between target variables and constructing the CTs. Some recent works used the linear correlation coefficient to explain target dependencies [21, 22]. Other measures could be employed for the same purpose, leading to different representations and predictive performances. In our experiments, we chose to use the Random Forest variable importance $\left(R F_{i m p}\right)$. This choice was based on the fact that this metric was reported as a reliable feature selector, which explores the impact of each variable on the whole Random Forest ensemble performance [12]. Furthermore, $R F_{i m p}$ does not suppose a linear behaviour between inputs and outputs of a predictive task, making it nonlinear. It also reports variables with no contribution or that disrupted a target estimation within a regression model. These variables are not considered then building CTs. For more details about the $R F_{i m p}$ calculation, please refer to Section 4.2.1.

In many cases, all outcomes can offer some degree of contribution to explaining other targets. Considering that MOTC aims at reducing the number of trained regression models, it must only select the most relevant outputs during the construction of the CT. For this task, we propose the use of the Hoeffding Bound (HB) to define an interval where the evaluated target dependencies are more relevant.

The Hoeffding Bound theorem [13] states that a variable $v$ whose range is $R$, which was independently observed $n$ times having a mean $\bar{v}$, has a true mean of at least $\bar{v}-\epsilon$ with statistical probability $1-\delta$ (the error probability stated), where:

$\epsilon=\sqrt{\frac{R^{2} \ln \left(\frac{1}{\delta}\right)}{2 n} .}$

To limit the ramification factor of each split, only targets whose importance fluctuate around the true importance of the best feature are selected. In this sense, assuming that the observed importance of the best feature is $\overline{i m p_{\mathrm{bt}}}$, we only select targets with importance greater than $\overline{i m p_{\mathrm{bt}}}-\epsilon$.

Algorithm 3 presents the general MOTC approach. We defined the default maximum depth for CTs $\left(\operatorname{Max}_{\text {depth }}\right)$ as $2 \times \log _{2}(d)$ if $d<6$, and $\log _{2}(d)$ otherwise, being $d$ the number of target variables. Empirically, we found these values as a compromise between predictive performance and complexity reduction. In the algorithm, $X$ and $Y$ represent the input and output variables respectively; $h b$ is the calculated Hoeffding Bound, and Max ${ }_{\text {depth }}$ is the parameter for controlling the maximum depth of the CT generated. Lastly, the procedure importance calculates the statistical dependencies between targets, in our case, it uses the $R F_{\text {imp }}$ measure.

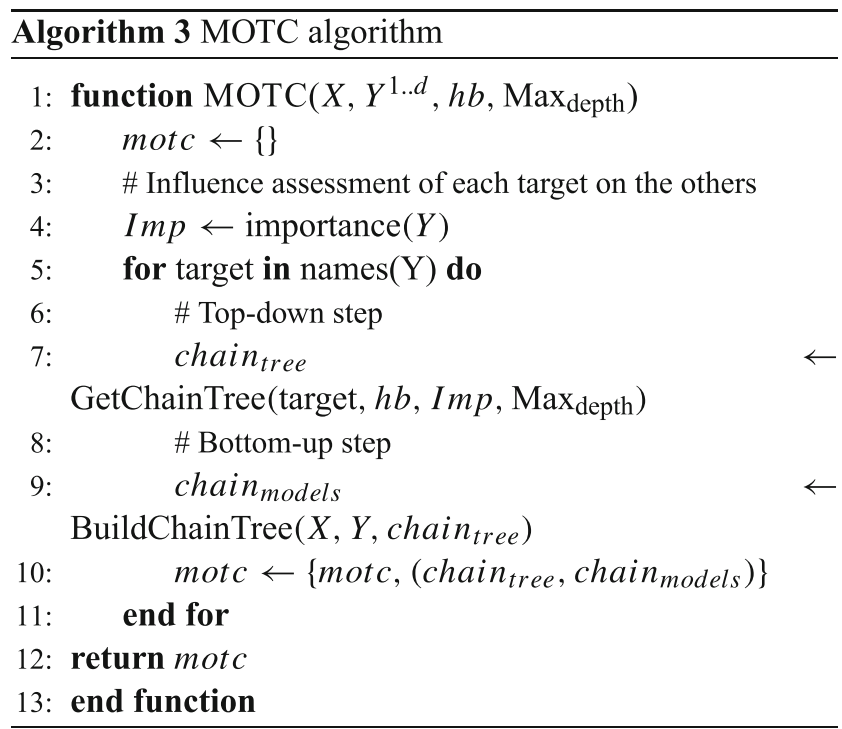

The top-down building procedure of a CT, presented in Algorithm 4, can also be set to ignore the $\mathrm{HB}$ when defining the tree structure. This fact is treated in line 5 of Algorithm 4. If one configures MOTC to perform this, branch factor will be greater since there is no target filtering. The resulting CTs will be larger, negatively impacting in the training time and memory costs. This was not done in our experiments, since computational complexity reduction was one of our goals. Although this evaluation is out of the scope of this work, it is possible to evaluate the use of a larger model to increase the predictive performance of a given MTR problem. The presented procedure starts with a tree with a single node, the root one, which corresponds to the desired target variable. The top-down step builds a tree structure hierarchy by adding the most relevant outputs for a target as its descendents, according to a relevance interval limited by the HB. The recursive callings of this procedure end when the maximum defined depth is reached. Algorithm 4 
receives the desired target for the CT building, $h b$ which corresponds to the Hoeffding Bound, Imp which is related to the outputs importance values, and the maximum depth for the CT, Max depth.

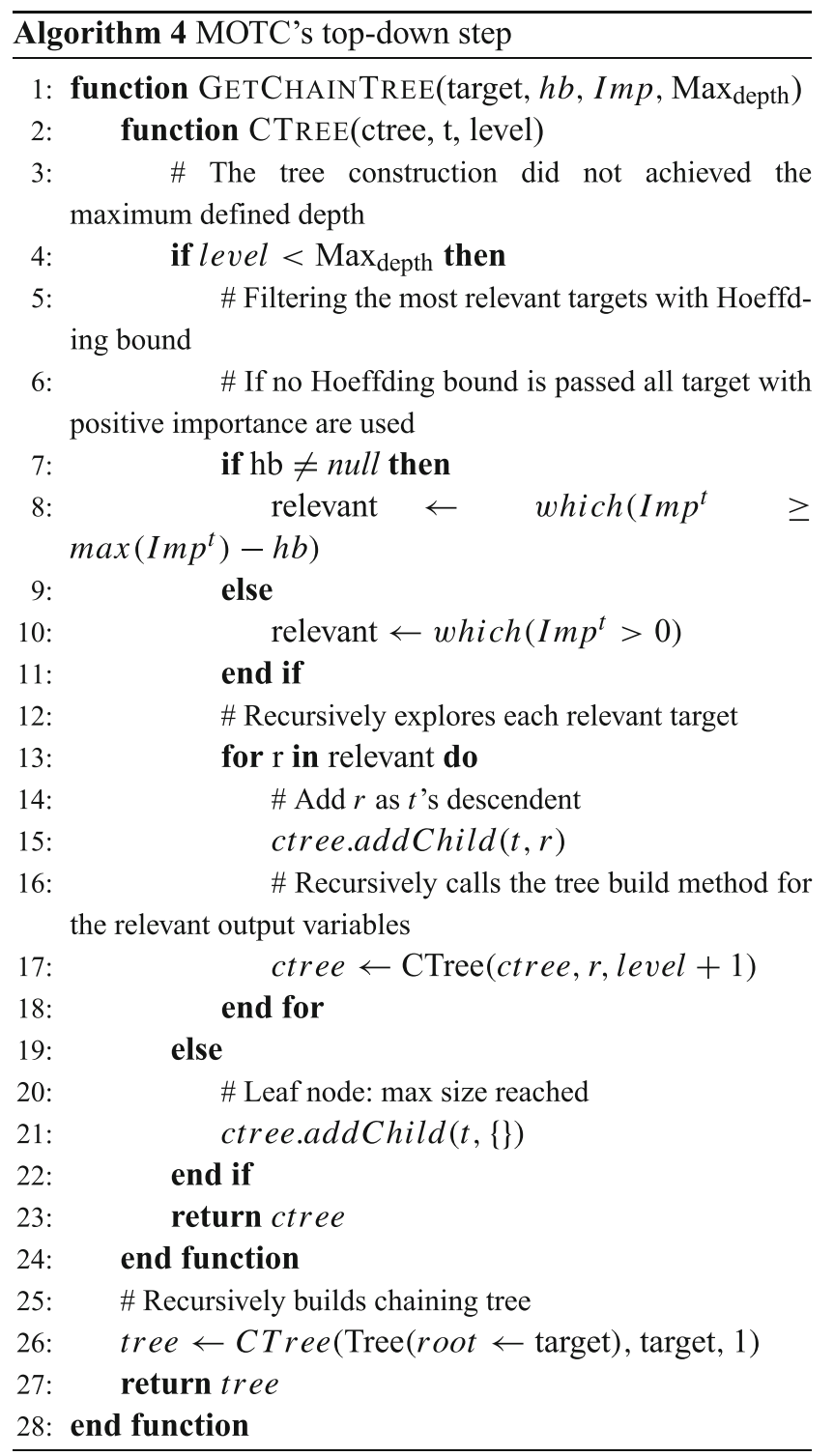

Lastly, we present the pseudo-code for the bottomup procedure done by MOTC in Algorithm 5. This step recursively travels a CT until it reaches the deepest nodes. After that, regression models for the leaves are constructed with only the original attributes. Then, the same operation is done while moving up in the tree. The inner nodes, including the tree root, depend on the predictions of the lower models, i.e, each model stacks the predictions of its descendents as new input features. In this sense, the bottom-up procedure assigns a regressor for each node in the CT. The MOTC's bottom-up procedure receives as parameters $X$ and $Y$, which correspond to the input and output variables, respectively, and the previous built $\mathrm{CT}$, chain $_{\text {tree }}$.

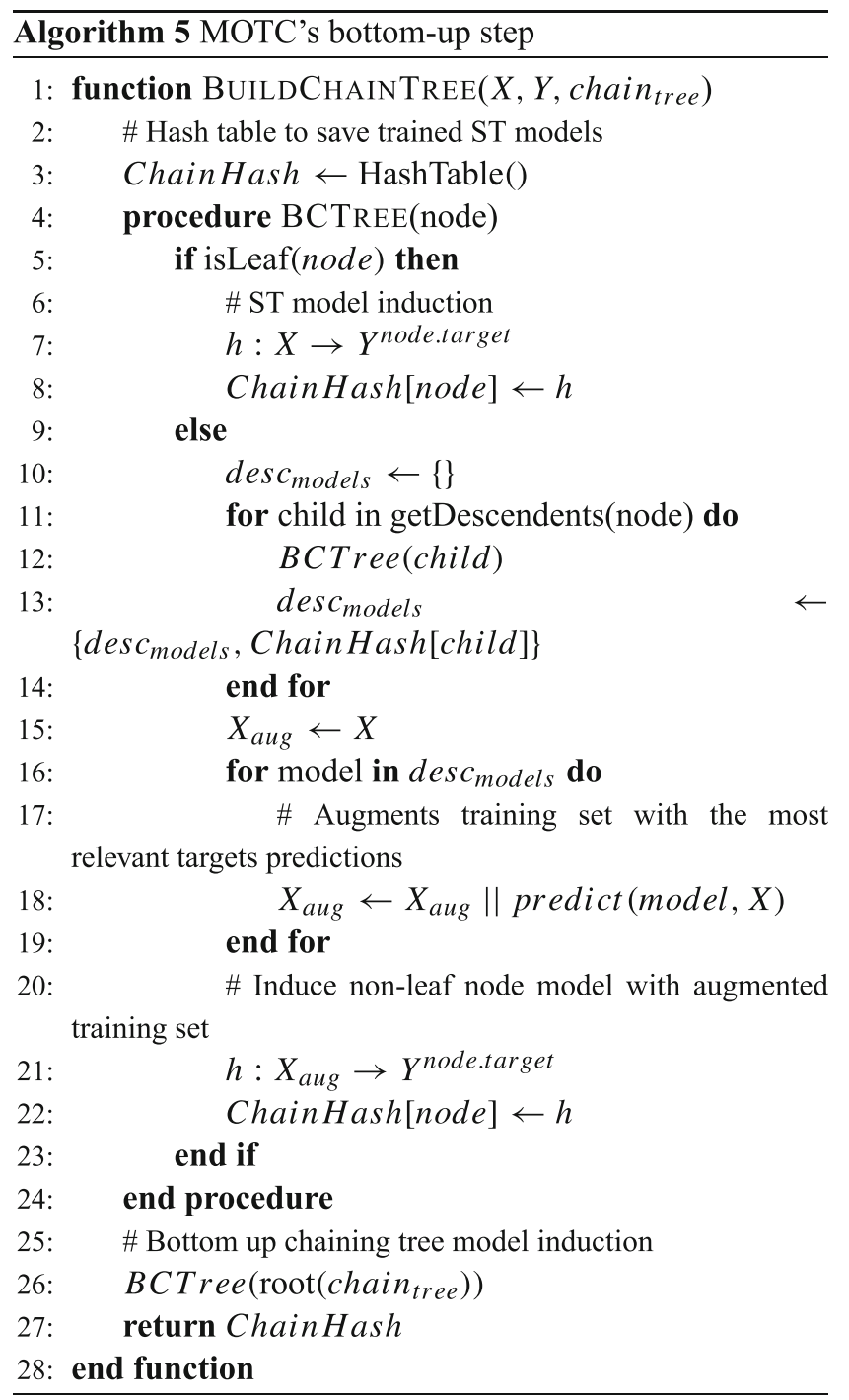

It is worth highlighting that since all leaves in a target CT correspond to ST models without augmented training sets, these regressors are shared between all CTs. In this sense, we avoid redundant training of models, which speeds up computation while also preventing memory waste.

\section{Materials and Method}

This section describes the experimental setup of this work. Initially, it presents the datasets explored. It also exposes the regression techniques and the motivation of their choice. Lastly, the metrics to evaluate the results are presented. 


\subsection{Datasets}

To evaluate the proposed algorithm, 18 MTR benchmark datasets were selected. ${ }^{1}$ Table 4, in Appendix A, characterises the datasets regarding the number of samples, input and output variables and also provides a brief description of their content as explored in [19, 25].

\subsection{Regression Techniques}

In this subsection, we present the two regression techniques employed to build the models: Support Vector Machines (SVM) and Random Forest (RF). We decided to use these algorithms due to their different strategies and wide applications. RF is an ensemble technique where multiple decision trees are combined under the idea of bagging, whereas SVMs relies on statistics foundations to fit a hyperplane able to perform classification and regression. In this research, we used the implementations of the $\mathrm{R}$ packages ranger and e1071 for RF and SVM, respectively, along with their default parameter settings.

\subsubsection{Random Forest}

Originally proposed by Breiman [3], RF is an ensemble algorithm used for both classification and regression tasks. In ensembles algorithms, different models with multiple approaches are employed and, in the case of RFs in specific, decision trees are combined in the form of a forest.

Initially, the algorithm bootstraps by sampling instances with replacement and selecting a subset of features. Following, the subset is used to build a decision tree, repeating this same procedure until a predetermined number of trees are created. After that, in order to perform either classification or regression, all trees are used in conjunction. According to Breiman [3], RF is robust to noise and the decision trees' feature selection capacities are naturally inherited. Moreover, its ensemble properties guarantees no overfitting.

Another main advantage of RFs consists of being able to measure attributes' importance $\left(R F_{\mathrm{imp}}\right)$. In order to do so, for each sampling performed, about a third of the training set is left aside (out-of-bag examples). Next, out-of-bag examples' attributes are randomly permuted, and tested by the forest. Permutations that increase the RF error can point out which attribute is more relevant. Hence, measuring its importance to the task.

For instance, if the error is increased by inserting noise (permutation) on a specific attribute, such attribute is likely

\footnotetext{
$\overline{{ }^{1} \text { http://mulan.sourceforge.net/datasets-mtr.html }}$
}

to be critical. The same applies for the opposite situation. In this sense, the $R F_{\text {imp }}$ of each variable is calculated by comparing the variation in the ensemble's prediction error obtained before and after the random permutation. If there is no alteration, it implies that the attribute does not contribute to the prediction task. In the same manner, if the error is reduced, the attribute is hindering $\mathrm{RF}$ predictions.

For regression, RFs take each trained tree to predict continuous values. In the majority of implementations, the final prediction is given by the mean value predicted by all trees in the forest.

\subsubsection{Support Vector Machine}

Support Vector Machine is a well established method for signal processing tasks. It was first introduced in Cortes and Vapnik [6] as a classification model for binary problems. SVM objective consists of creating an optimal separation hyperplane that splits the data into two classes. Such hyperplane's boundaries are defined by points referred as support vectors, hence naming the algorithm.

In situations whose data are not linearly separable, kernel tricks are employed. Such operation maps the original data into a higher dimensional space where the problem is more likely to be solvable.

Since we are interested in SVM for regression, we employed Support Vector Regression (SVR) [9]. In SVR, a hyperplane, defined by the Eq. 2, is built by minimising the Eq. 3 subject to Eq. 4.

$$
\langle\mathbf{w}, \mathbf{x}\rangle-b=0
$$

$\frac{1}{2}\|\mathbf{w}\|^{2}$

$\left\{\begin{array}{l}y_{i}-\langle\mathbf{w}, \mathbf{x}\rangle-b \leq \epsilon \\ \langle\mathbf{w}, \mathbf{x}\rangle+b-y_{i} \leq \epsilon\end{array}\right.$

In these Equations, $\mathbf{w}$ corresponds to the normal vector to the hyperplane, $x_{i}$ is a training sample with label $y_{i}$ and $\epsilon$ is a threshold value for the margin. Hence, all predictions, given by $\langle\mathbf{w}, \mathbf{x}\rangle+b$, must be within the range implied by $\epsilon$. Furthermore, finding the optimal hyperplane is mapped to a convex problem with a feasible solution. Nonetheless, in situations with no feasible solutions, an extra variable $\xi$ may be added to cope with such constraints.

\subsection{Performance Evaluation}

Aiming at evaluating the models created during the experiments, we used two different metrics: the average 
Relative Root Mean Squared Error (aRRMSE) and the Counting of Trained Regression Models (CTRM). Also, the MTR methods were performed using a 10-fold crossvalidation strategy to minimise bias.

The aRRMSE is obtained by averaging the RRMSE (Relative Root Mean Squared Error) calculated for each target variable. RRMSE measures a model error decrease over a naive predictor which always outputs the target mean value. The latter servers a baseline in the metric and allows the measurement of the improvement over a shallow predictor, being used in various MTR works [2, 20$23,25]$ to compare non-homogeneous targets distributions. The aRRMSE calculation is presented in Eq. 5, where $d$ represents the number of targets variables, $N$ the number of testing instances, $y, \hat{y}$ and $\bar{y}$ denote, respectively, the real value of the output, its predicted and mean values.

$$
\operatorname{aRRMSE}=\frac{1}{d} \sum_{t=1}^{d} \sqrt{\frac{\sum_{k=1}^{N}\left(y_{t}^{k}-\hat{y}_{t}^{k}\right)^{2}}{\sum_{k=1}^{N}\left(y_{t}^{k}-\bar{y}_{t}\right)^{2}}}
$$

As the time and memory costs of the MTR local methods directly depends on the employed regressor, we defined the Counting of Trained Regression Models (CTRM) metric to compare how much computational resources they used. CTRM is defined as the total number of ST models induced by an MTR method in a dataset. This metric can be employed as a measure of how much memory each MTR approach uses. In other words, the smaller the CTRM value of an approach, the more memory efficient the evaluated MTR method is. Additionally, training fewer models results in less time complexity of the solution.

Both aRRMSE and CTRM support the comparison of possible method superiority through the application of the Friedman statistical test and the Nemenyi post hoc test with a Critical Difference (CD) diagram, as previously proposed in [7]. They are used to evaluate if the null hypothesis is rejected. The null hypothesis states that the performances of the MTR methods are equivalent regarding the evaluated metric. In this sense, when the null hypothesis is false, we can apply the Nemenyi post hoc test, which states that the performance of two distinct models are significantly different if the corresponding average ranks differ by at least a $\mathrm{CD}$ value.

We also defined the Relative Performance (RP) and Relative Size (RS) measures, derived from aRRMSE and CTRM, respectively. RP and RS are used for evaluating how MOTC compares against ERC in predictive performance and memory efficiency since both methods define chained regression models. RP measures the reduction in error of an MTR approach over another, whereas RS measures how time and memory conservative an MTR method A is when compared with another method B. The RP of a method $A$ against another method $B$ is given according to Eq. 6 .

$R P_{B \mid A}=\frac{\mathrm{aRRMSE}_{A}}{\mathrm{aRRMSE}_{B}}$

If the obtained RP is equal to one, there is no performance difference between the compared algorithms. An RP greater than one means that $\mathrm{B}$ is better than $\mathrm{A}$ in the evaluated problem. The same applies to the opposite.

$\mathrm{RS}$ calculation is given in Eq. 7. An RS greater than one shows that $\mathrm{B}$ spends more regressor models than $\mathrm{A}$. Moreover, an obtained $R S=0.5$ implies that $\mathrm{B}$ spent half the number of models used by $\mathrm{A}$.

$R S_{B \mid A}=\frac{\mathrm{CTRM}_{A}}{\mathrm{CTRM}_{B}}$

Lastly, aiming to evaluate the inter-target dependency, we comprised all generated CTs into a single representation. For each dataset, the obtained CTs considering all targets and cross-validation folds were merged into an oriented graph. Every time there was a relation of the type " $B$ explains A", being A and B target variables, the edge weight connecting the two targets was incremented. So, in the obtained graphs the edges represent how many times an output was used to explain another. The resulting structures were called Condensed CT Graphs (CCTG).

\section{Results and Discussion}

In this section, the comparative results between MOTC, simple ST, and the other MTR approaches (MTRS and ERC) are presented. First, we discuss the results regarding aRRMSE. Then, we discuss how many regression models were trained for each method, while also presenting statistical comparisons concerning both predictive performance and number of induced models. Lastly, our method is compared against ERC, which also uses chained regressors. We show that MOTC had a similar predictive performance compared to ERC, employing fewer regression models in the process. We also present the time complexity analysis for all compared MTR methods. Likewise, some insights on how MOTC could be employed to better comprehend the relationships between targets in an MTR problem are also presented.

\subsection{Predictive Performance}

Table 1 reports the obtained aRRMSE regarding all MTR methods and base regression techniques ${ }^{2}$, per benchmark

\footnotetext{
${ }^{2}$ The source codes for MOTC and the other evaluated MTR methods are disponible in http://www.uel.br/grupo-pesquisa/remid/? page_id=145.
} 
Table 1 Average Relative Root Mean Squared Error (aRRMSE) results considering all datasets.

\begin{tabular}{|c|c|c|c|c|c|}
\hline Dataset & Algorithm & ST & MTRS & ERC & MOTC \\
\hline \multirow[t]{2}{*}{ ATP1D } & $\mathrm{RF}$ & 0.3919 & 0.3904 & 0.3902 & 0.3910 \\
\hline & SVM & 0.4396 & 0.4402 & 0.4398 & 0.4398 \\
\hline \multirow[t]{2}{*}{ ATP7D } & $\mathrm{RF}$ & 0.5164 & 0.5169 & 0.5178 & 0.5187 \\
\hline & SVM & 0.6404 & 0.6414 & 0.6410 & 0.6408 \\
\hline \multirow[t]{2}{*}{ OES97 } & $\mathrm{RF}$ & 0.5164 & 0.5135 & 0.5133 & 0.5153 \\
\hline & SVM & 0.6118 & 0.6123 & 0.6109 & 0.6116 \\
\hline \multirow[t]{2}{*}{ OES10 } & $\mathrm{RF}$ & 0.4070 & 0.4081 & 0.4070 & 0.4073 \\
\hline & SVM & 0.5464 & 0.5456 & 0.5451 & 0.5464 \\
\hline \multirow[t]{2}{*}{ RF1 } & $\mathrm{RF}$ & 0.0782 & 0.0582 & 0.0731 & 0.0723 \\
\hline & SVM & 0.1215 & 0.1070 & 0.1151 & 0.1191 \\
\hline \multirow[t]{2}{*}{ RF2 } & $\mathrm{RF}$ & 0.0847 & 0.0784 & 0.0852 & 0.0872 \\
\hline & SVM & 0.1095 & 0.1064 & 0.1084 & 0.1091 \\
\hline \multirow[t]{2}{*}{ SCM1D } & $\mathrm{RF}$ & 0.2871 & 0.2757 & 0.2823 & 0.2823 \\
\hline & SVM & 0.3309 & 0.3232 & 0.3258 & 0.3260 \\
\hline \multirow[t]{2}{*}{ SCM20D } & $\mathrm{RF}$ & 0.3647 & 0.3313 & 0.3347 & 0.3277 \\
\hline & SVM & 0.3972 & 0.3522 & 0.3474 & 0.3472 \\
\hline \multirow[t]{2}{*}{ SDM } & $\mathrm{RF}$ & 0.6721 & 0.6631 & 0.6661 & 0.6620 \\
\hline & SVM & 0.7699 & 0.7714 & 0.7667 & 0.7654 \\
\hline \multirow[t]{2}{*}{ SF1 } & $\mathrm{RF}$ & 1.0051 & 1.1280 & 1.0161 & 1.0578 \\
\hline & SVM & 0.9390 & 0.9477 & 0.9250 & 0.9337 \\
\hline \multirow[t]{2}{*}{ SF2 } & $\mathrm{RF}$ & 0.8487 & 0.9410 & 0.8617 & 0.8729 \\
\hline & SVM & 0.7825 & 0.7787 & 0.7827 & 0.7843 \\
\hline \multirow[t]{2}{*}{ Jura } & $\mathrm{RF}$ & 0.6061 & 0.5974 & 0.5969 & 0.5971 \\
\hline & SVM & 0.6409 & 0.6413 & 0.6391 & 0.6418 \\
\hline \multirow[t]{2}{*}{ WQ } & $\mathrm{RF}$ & 0.9066 & 0.9392 & 0.9059 & 0.9139 \\
\hline & SVM & 0.9630 & 0.9535 & 0.9581 & 0.9617 \\
\hline \multirow[t]{2}{*}{ ENB } & $\mathrm{RF}$ & 0.1504 & 0.1173 & 0.1304 & 0.1206 \\
\hline & SVM & 0.2499 & 0.2173 & 0.2404 & 0.2322 \\
\hline \multirow[t]{2}{*}{ Slump } & $\mathrm{RF}$ & 0.8365 & 0.8325 & 0.8222 & 0.8497 \\
\hline & SVM & 0.6924 & 0.6862 & 0.6818 & 0.7056 \\
\hline \multirow[t]{2}{*}{ Andro } & $\mathrm{RF}$ & 0.7941 & 0.7349 & 0.7614 & 0.7734 \\
\hline & SVM & 1.1348 & 0.9243 & 1.0089 & 1.0208 \\
\hline \multirow[t]{2}{*}{ OSALES } & $\mathrm{RF}$ & 0.7577 & 0.7275 & 0.7332 & 0.7455 \\
\hline & SVM & 1.1726 & 1.1685 & 1.1702 & 1.1716 \\
\hline \multirow[t]{2}{*}{ SCPF } & $\mathrm{RF}$ & 0.9263 & 0.9387 & 0.8778 & 0.8711 \\
\hline & SVM & 0.8242 & 0.8256 & 0.8151 & 0.8159 \\
\hline
\end{tabular}

dataset. The columns ST, MTRS, ERC and MOTC represent the MTR approaches, while the base regressors are presented in column Algorithm, resulting in a base regressorMTR approach combination by line. The highlighted value of aRRMSE corresponds to the better performing algorithm/MTR approach pair for that dataset.

MTRS performed better in 13 algorithm-MTR approach combinations (six cases using RF and the remaining with
SVM as base regressor). ERC achieved the best prediction errors in 11 cases (six times when using RF and five with SVM). ST got the best aRRMSE in seven cases (four with the RF and three with SVM). MOTC, obtained the best results in five cases (three times combined with RF and two cases with SVM). Although our approach was out-performed by the others, the performance difference is minimal, being very close to ERC in the majority of the cases. Nonetheless, MOTC outperforms ERC, which is also based on chained regressors, by a large margin when considering memory and time, as discussed further in this work. Also, our method offers a highly representative model of how targets are correlated to each other. None of the compared approaches (ST, MTRS and ERC) can be used to explain the existing dependencies between the output variables.

\subsection{Number of Induced Models}

We report the mean CTRM value for each MTR method in Table 2 . This analysis is particularly relevant, since the number of generated models directly impacts the obtained solution's time and memory costs. Since one of our goals was to obtain a memory and time conservative method, without compromising prediction performance, this analysis is a core part of this paper.

Table 2 Mean Counting of Trained Regression Models (CTRM) obtained by each method in all evaluated datasets.

\begin{tabular}{llllll}
\hline Dataset & ST & MTRS & ERC & MOTC (RF) & MOTC (SVM) \\
\hline ATP1D & 6 & 12 & 60 & 30 & 30 \\
ATP7D & 6 & 12 & 60 & 39.9 & 37.9 \\
OES97 & 16 & 32 & 160 & 84.8 & 87.1 \\
OES10 & 16 & 32 & 160 & 84.1 & 81.8 \\
RF1 & 8 & 16 & 80 & 24.3 & 24.3 \\
RF2 & 8 & 16 & 80 & 24.3 & 24.3 \\
SCM1D & 16 & 32 & 160 & 64 & 64.5 \\
SCM20D & 16 & 32 & 160 & 65.2 & 65.2 \\
EDM & 2 & 4 & 4 & 4 & 4 \\
SF1 & 3 & 6 & 18 & 8.6 & 8.4 \\
SF2 & 3 & 6 & 18 & 7.3 & 7.3 \\
Jura & 3 & 6 & 18 & 9.1 & 9.1 \\
WQ & 14 & 28 & 140 & 64 & 63.5 \\
ENB & 2 & 4 & 4 & 4 & 4 \\
Slump & 3 & 6 & 18 & 9.4 & 9.4 \\
Andro & 6 & 12 & 60 & 30.6 & 30 \\
OSALES & 12 & 24 & 120 & 51.5 & 52.6 \\
SCFP & 3 & 6 & 18 & 9 & 9 \\
\hline
\end{tabular}


ST, as the simplest approach, induces as many regressor models as the number of problem targets. MTRS always generates twice the number of ST models. In its turn, ERC creates the maximum number of combinations of targets if the number of outputs is lesser than three. Otherwise, ten random combinations are chosen, so CTRM is equal to ten times the number of target variables. MOTC adaptively chooses the number of trained predictors, which is equal to the summed number of nodes in all generated CTs. The choice of MOTC's Max depth parameter and the application of HB directly impact the obtained CTRM.

As expected, $\mathrm{ST}$ is the most time and memory conservative approach, followed by MTRS in all cases except when the number of targets is equal to two. In these cases, both ERC and MOTC are tied with MTRS. MOTC is the third most conservative approach, independently of the chosen regressor, and at the least position comes ERC. Despite employing more models than the ST and MTRS approaches, MOTC dynamically adapts its CT structures for each problem offering insights on how the targets influence each other. This fact offers a useful tool for experts and non-experts when dealing with MTR tasks.

The slight different CTRM obtained by MOTC when using RF or SVM as base regressors is a result of the target importance metric choice. We calculated $R F_{i m p}$ upon a set of RF models trained using only the target variables. RF's variable importance value takes into consideration the increase in ensemble's predictive error brought by random permuting the values of an explaining feature. Also, each tree in a RF is constructed by sampling training instances and subsetting features at random. In fact, there are many aspects of randomness which result in slightly different CTs, depending on the number of elements and targets of the problem, as well as the number of trees used to calculate $R F_{\text {imp }}$.
The authors encourage the evaluation of the impact brought by using different importance metrics and values for CT's Max ${ }_{\text {depth }}$ on the obtained CTRM and aRRMSE. This analysis, however, is out of the scope of this work.

\subsection{Statistical Comparison}

We performed the Friedman test on the predictive performance of the compared MTR methods. Figure 2 reports the Nemenyi post test ranked comparison considering the predictive performance obtained over all datasets. Lower rankings mean better predictive performance (with $\mathrm{CD}=$ 0.7837 and $\alpha=0.05)$. The obtained Friedman test's p-value $(<2.2 e-16)$ confirm there were statistical differences between compared MTR methods and employed regressors, regarding the obtained aRRMSE.

As shown in Fig. 2, the first connected group comprehends to ERC, MTRS and MOTC using RF as the base regressor model. The second group comprehends MOTC and ST, again along with RF. In this sense, there is no statistical difference between ERC, MTRS and MOTC. In the third connected group, corresponding to using SVM as regressor base model, MTRS appears at the first position, followed by ERC, MOTC and ST. Again, no statistical difference was observed between ERC, MTRS and MOTC. Using both RF and SVM, MOTC is connected with ST. It seems that the chosen base regression algorithm can impact in the ranking of the selected MTR methods. Besides, the distance observed between MTR approaches was lesser when using RF, which was related to the greater generalisation capacity commonly achieved by ensemble methods.

As discussed in Section 5.4, MOTC performed very comparably to ERC in almost all cases. This fact is related to their similar resulting models' structure. In most of the
Figure 2 Nemenyi post test resulting, considering the predictive performance over all datasets.

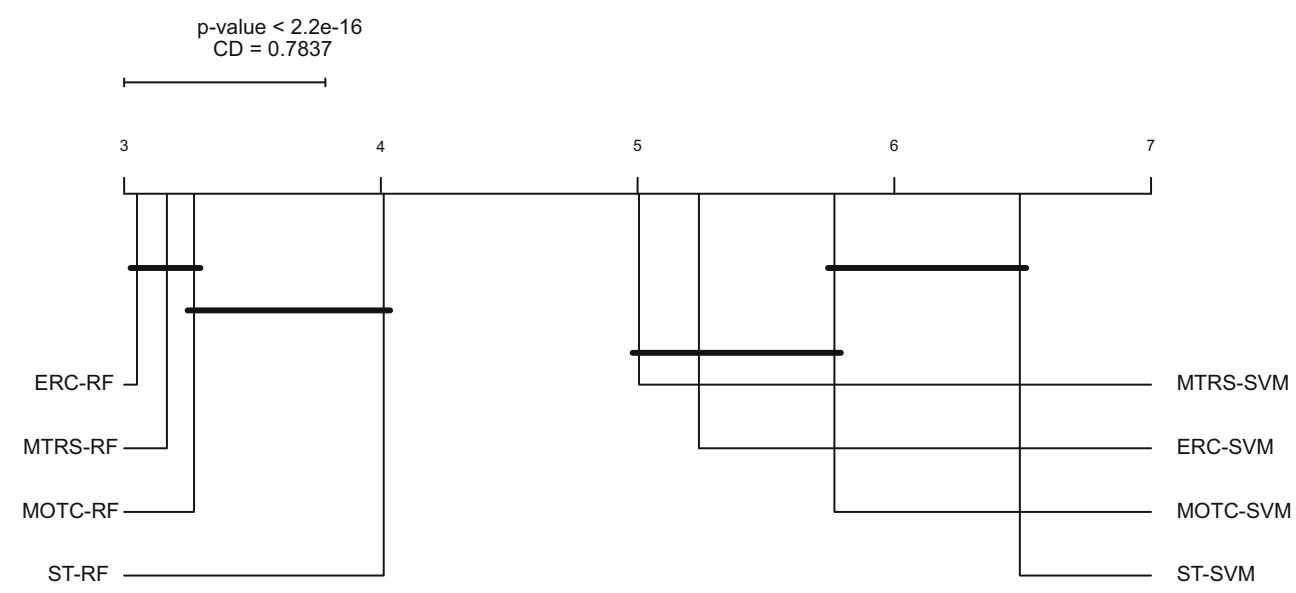


cases, MOTC was equivalent or obtained slightly greater error than ERC. For this reason, when MTRS outperformed ERC, it also was superior to MOTC. On another hand, when ERC achieved the least aRRMSE, MOTC results were very close to it, but still somewhat worse. Thus, MOTC appears at third position as a reflex the few cases it outperformed both ERC and MTRS.

We present the statistical comparison regarding the number of generated models per MTR method (at $C D=$ 0.4555 and $\alpha=0.05$ ) in Fig. 3. As expected, ST provides the most conservative approach, being at the first position in all cases. MTRS comes in second place, spending twice the ST number of models. MOTC is the third more conservative approach, independently of the chosen regressor. As expected, ERC is the worst method, expending a lot more regression models than the other MTR approaches. This scenario could be even worse if one chooses to remove the number of generated chains limitation, proposed in original ERC formulation (maximum number of random permutations equal to ten). It is worth mentioning that all methods were statistically different among themselves in this test.

We also would like to emphasise that MOTC adaptively generates a different number of models by analysing how the problem targets relate to each other. All compared models (ST, MTRS and ERC) produce a fixed number of regression models, which are not easy to interpret in the inter-target relationship context. In contrast, MOTC offers clear information about how an output helps to explain the distribution of the other targets. Further, by using the CTs from MOTC, it is possible to compare their results with prior knowledge about a research field and even discover new interesting properties and inferences from the targets.
Table 3 Relative Performance (RP) and Relative Size (RS) when comparing MOTC with ERC in all datasets.

\begin{tabular}{llllll}
\hline \multirow{2}{*}{ Dataset } & \multicolumn{2}{l}{$\mathrm{RP}_{\text {MOTC|ERC }}$} & & \multicolumn{2}{l}{$\mathrm{RS}_{\text {MOTC|ERC }}$} \\
\cline { 2 - 3 } \cline { 5 - 6 } & $\mathrm{RF}$ & $\mathrm{SVM}$ & & $\mathrm{R}$ & $\mathrm{R}$ \\
\hline ATP1D & 1.00 & 1.00 & & 0.50 & 0.50 \\
ATP7D & 1.00 & 1.00 & & 0.67 & 0.63 \\
OES97 & 1.00 & 1.00 & & 0.53 & 0.54 \\
OES10 & 1.00 & 1.00 & & 0.53 & 0.51 \\
RF1 & 1.01 & 0.96 & & 0.30 & 0.30 \\
RF2 & 0.95 & 0.99 & & 0.30 & 0.30 \\
SCM1D & 1.00 & 1.00 & & 0.40 & 0.40 \\
SCM20D & 1.02 & 1.00 & & 0.41 & 0.41 \\
EDM & 1.01 & 1.00 & & 1.00 & 1.00 \\
SF1 & 0.97 & 0.99 & & 0.48 & 0.47 \\
SF2 & 0.99 & 1.00 & & 0.41 & 0.41 \\
Jura & 1.00 & 1.00 & & 0.51 & 0.51 \\
WQ & 0.99 & 1.00 & & 0.46 & 0.45 \\
ENB & 1.09 & 1.03 & 1.00 & 1.00 \\
Slump & 0.97 & 0.97 & 0.52 & 0.52 \\
Andro & 1.02 & 1.00 & 0.51 & 0.50 \\
OSALES & 0.98 & 1.00 & 0.43 & 0.44 \\
SCFP & 1.01 & 1.00 & 0.50 & 0.50 \\
\hline & & & &
\end{tabular}

\subsection{MOTC and ERC Comparison}

The idea of chaining regressor models is shared by MOTC and ERC, but the second does this action in a naive strategy. Table 3 presents the obtained RP and RS when comparing MOTC with ERC.
Figure 3 Nemenyi post test resulting, considering the generated number of models in all datasets.

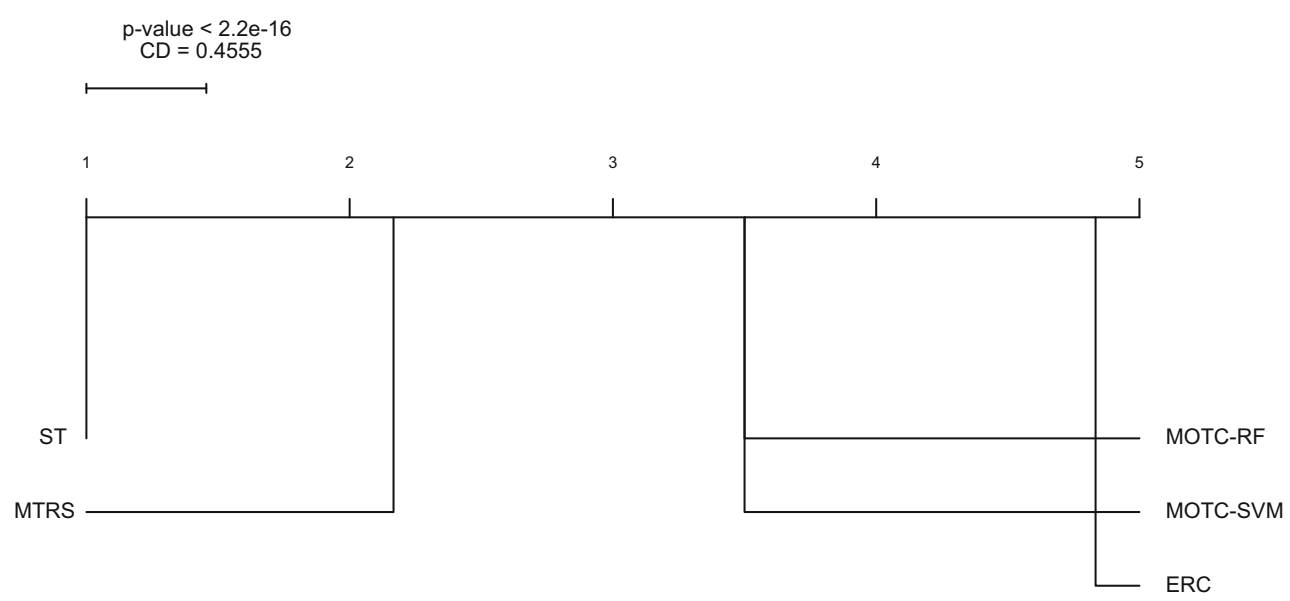


MOTC got RP values very close to ERC in the majority of datasets. In the worst case, MOTC achieved $95 \%$ of ERC's performance; in some cases our approach stood out.

On the other hand, when it comes to RM, MOTC was prominently superior to ERC. There were only two cases where MOTC generated as many models as ERC. They correspond to datasets EDM and ENB, which have only two target variables. Excluding these simpler problems, in the worst case, MOTC built $65 \%$ fewer models than ERC. In fact, in most of the time, MOTC used about half regressors than ERC did.

Therefore, our proposed approach obtained a competitive predictive performance when compared with ERC. Furthermore, it combines modest time and memory costs for modelling through an informative and easy to interpret structure.

\subsection{Complexity Analysis}

Consider $d$ as the number of problem targets and $b$, the complexity of the chosen base regressor. ST induces as many models as the number of outputs, so its time complexity is $\mathcal{O}(d \times b)$. MTRS, in its turn, produces twice the number of ST's models. For that reason, MTRS's time complexity is $\mathcal{O}(2 \times d \times b)$, which does not asymptotically differ from ST. ERC, without limiting settings, produces as many regressors as the maximum number of targets permutations. In this sense, ERC's complexity would be $\mathcal{O}\left(d^{2} \times(d-1) ! \times b\right)$. However, by following the ERC's authors recommendation of generating only ten random permutations if the number of targets is greater than three, the obtained complexity is $\mathcal{O}(10 \times d \times b)$.

MOTC generates regression models with a tree fashion. The growing of new nodes in the produced CTs depends on two main factors: the max tree depth constraint (parameter $\left.\max _{\text {depth }}\right)$ and the branching factor. The latter is determined by using or not the HBs. Suppose $\zeta$ to be the summed number of nodes contained in all targets CTs, excluding the redundant leaf nodes, as previously discussed in Section 3. MOTC time complexity is $\mathcal{O}(\zeta \times b)$. Although there is no asymptotic difference between MOTC and constrained ERC version, our experiments proved that the former method is the most time and memory efficient.

Also, when the number of targets is sufficiently small, MOTC generates as many models as MTRS or ERC. In fact, if the max $x_{\text {depth }}$ is setted to one, MOTC will mimic MTRS. Moreover, if MOTC branching factor get too close to $d$ and/or the maximum depth of CTs is sufficiently large, our method should generate more regression models than ERC. Nevertheless, as our experiments demonstrated,
MOTC with its default parameters is capable of achieving comparable performance results while being more time and memory conservative than ERC.

\subsection{Analysis of the Relationship Between Targets with CTs}

The generated CCTGs allow us to get some interesting information. For example, one could analyse that an output almost always helps to explain another, but the opposite is not true. Also, the presence of connections among targets with small edge values is an indication of little influence of a target on another. Additionally, cycles inside the graphs could be detected, meaning there are indirect relationships among targets. Lastly, different groups of dependency could be detected, resulting in decomposing a MTR target into smaller multi-output tasks.

All obtained CCTGs are presented in Appendix B "Obtained condensed chaining tree graphs and target labels". For the matter of readability, in all graphs, the target names were substituted by $T$ followed by an output identifier. One could check their original names by referring to Tables 5 to 11 .

We present some real examples about the mentioned CCTG behaviour. Some cases were pretty straightforward, presenting mutual dependencies in all cases, like in datasets EDM and ENB (Fig. 4). They represent the two datasets with the smallest number of target variables, so it is to be expected a simple inter-target relationship.

Other interesting cases were discovered when analysing the datasets SCPF and Slump (Fig. 5). In SCPF, it is possible to observe that there are no dependencies between targets $\mathrm{T} 2$ (num_views) and T1 (num_comments). Meanwhile, both of them influence and are influenced by T3 (num_votes). As highlighted in Section 4.1, SCPF evaluates the number of votes, views and comments obtained by certain online issues within a period. Our analysis shows that the number of votes that an issue receives depends on its number of views and comments, which seems reasonable in the context of online content resources. However, an issue could achieve high rates of views and comments, whereas not being voted.

The Slump dataset concerns the prediction of three properties of concrete (slump, flow and compressive strength). As shown in Fig. 5, there are mutual relationships between targets T2 (Flow) and T3 (Slump), and both of them influence the Compressive Strength (T1) of concrete. However, they are not influenced by T1. MOTCs takes advantage of not inserting $\mathrm{T} 1$ as additional prediction feature when modelling $\mathrm{T} 2$ and $\mathrm{T} 3$. 
In addition, another behaviour was observed when combining the generated CTs. There were cases where the targets were grouped regarding their inter-dependencies. In these situations, targets within a group did not influence outputs outside it. In this sense, this behaviour indicated that an original MTR task could be decomposed into smaller problems. The creation of separate target groups was observed in datasets andro, ATP1D (Fig. 6), SCM1D and SCM20D (Fig. 11).

Take as an example the dataset ATP1D. It concerns the prediction of the minimum air ticket price in the next day, given an observed date. ATP1D targets correspond to the expected minimum price for: (1) any airline with any number of stops, (2) any airline non-stop only, (3) Delta Airlines, (4) Continental Airlines, (5) Airtrain Airlines, and (6) United Airlines. Figure 6 give us pieces of evidence that the next day minimum price for non-stop airlines have influenced and was influenced by the price of Continental Airlines. The same comparison applies when comparing Delta and Airtrain Airlines, and United Airlines with flights with any number of stops. On the other hand, no influence was observed among different groups, considering all generated CTs and cross-validation data partitions.

Furthermore, complex patterns of relationships were observed in most of the datasets whose number of outputs are greater than eight. As the number of targets has increased, also has risen the diversity of the dependencies between the output variables. In datasets OES10 (Fig. 7), OES97 (Fig. 8), RF1, RF2 (Fig. 10), OSALES (Fig. 9), and WQ (Fig. 12), diverse inter-target dependence patterns were observed. In these datasets, most of the previously discussed cases of influence can be seen. In contrast, there were clear and simple patterns of relationship among targets in datasets SCM1D and SCM20D (Fig. 11), as previously presented. Thus, there is no direct association between the number of outputs in a predictive problem and the complexity level of the statistical dependencies between targets.

Lastly, by using a CCTG representation, one could apply a threshold value to consider the most prominent cases of target influence. In this way, by removing edges with small weights, the complex relationship between outputs could be decomposed into simpler MTR tasks or even separate ST problems. In fact, in many cases the weights in edges were unbalanced. For instance, a proper thresholding scheme would separate targets T1 and T4 into a separate MTR problem, when considering datasets RF1 and RF2 (Fig. 10). The RF datasets deal with the prediction of the Mississipi river flow in eight different sites. The strong dependency between sites CHSI2_48H_0 (T1) and EADM7_48H_0 (T4) should reflect some physical, geological and positional aspects which relate these two measured locals.

\section{Conclusion}

This paper proposed a new method, called Multi-output Tree Chaining (MOTC), for solving multi-target regression problems. Our solution describes the relationships between target variables as a tree structure toward a CT. In this structure, each node represents an output, and its children denote the targets which most explain it. CTs take into consideration a target relevance measure to assess the dependencies between outputs. We employed the Random Forest variable importance metric as a nonlinear descriptor of inter-target relations. Also, when constructing the CTs, aiming at limiting their ramification factor, we employed the Hoeffding Bound only to select the targets which most explain the targeted output. After building a CT for each target, regression models were inducted for each node, starting from the leaves. In MOTC, intermediate nodes use the original features of the problem augmented by the predictions that come from their descendants models.

An extensive experimental setup consisting in 18 standard multi-target benchmark datasets, combined with two regression techniques (Random Forest and Support Vector Machine), where we compared MOTC with three state-of-art MTR methods (ST, MTRS and ERC) was performed. The results showed that MOTC obtained competitive predictive performance. In fact, the performed statistical tests demonstrated that MOTC has no significant difference from MTRS and ERC methods concerning predictive performance, being superior to ST. It is worth mentioning that the choice of the used regression technique proved to impact in the final performance of the multitarget regression methods. This fact should be analysed in future works. Nevertheless, our method presented significant reduction over ERC regarding memory and time complexity.

Besides that, MOTC offers a comprehensive representation to analyse multi-target tasks, which none of the existing solutions present, enabling the verification of prior knowledge about a problem, and even the discovering of new properties.

Acknowledgements The authors would like to thank CAPES (Coordenação de Aperfeiçoamento de Pessoal de Nível Superior), $\mathrm{CNPq}$ (Conselho Nacional de Desenvolvimento Científico e Tecnológico) and FAPESP (Fundação de Amparo à Pesquisa do Estado de São Paulo) for financial support. 


\section{Appendix A: Datasets Used in the Experiments}

Table 4 Dataset's characteristics: dataset name, number of examples, number of input variables, number of targets, and description.

\begin{tabular}{|c|c|c|c|c|}
\hline Dataset & \#Examples & \#Input & \#Outputs & Description \\
\hline ATP1D & 337 & 411 & 6 & $\begin{array}{l}\text { Minimum air ticket price in the next day for } \\
\text { different options of companies and number of } \\
\text { stops. }\end{array}$ \\
\hline ATP7D & 296 & 411 & 6 & $\begin{array}{l}\text { Minimum air ticket price over the next seven days } \\
\text { for different options of companies and number of } \\
\text { stops. }\end{array}$ \\
\hline OES97 & 334 & 263 & 16 & $\begin{array}{l}\text { Occupational employment surveys performed in } \\
\text { 1997, containing the estimated number of employ- } \\
\text { ees in different jobs. }\end{array}$ \\
\hline OES10 & 403 & 298 & 16 & $\begin{array}{l}\text { Occupational employment surveys performed in } \\
2010 \text {, containing the estimated number of employ- } \\
\text { ees in different jobs. }\end{array}$ \\
\hline RF1 & 9125 & 64 & 8 & $\begin{array}{l}\text { River flows in the next } 48 \text { hours in eight different } \\
\text { rivers in the Mississippi River network. }\end{array}$ \\
\hline RF2 & 9125 & 576 & 8 & $\begin{array}{l}\text { River flows in the next } 48 \text { hours in eight different } \\
\text { rivers in the Mississippi River network with } \\
\text { precipitation forecast information added to the } \\
\text { input variables. }\end{array}$ \\
\hline SCM1D & 9803 & 280 & 16 & $\begin{array}{l}\text { Prices of different products in the next day in a } \\
\text { supply chain management. }\end{array}$ \\
\hline SCM20D & 8966 & 61 & 16 & $\begin{array}{l}\text { Mean price of different products over the next } 20 \\
\text { days in a supply chain management. }\end{array}$ \\
\hline EDM & 154 & 16 & 2 & $\begin{array}{l}\text { Parameter settings controlled by a human operator } \\
\text { during electrical discharge machining. }\end{array}$ \\
\hline SF1 & 323 & 10 & 3 & $\begin{array}{l}\text { Number of times common, moderate and severe } \\
\text { solar flares were observed in a } 24 \mathrm{~h} \text { interval. }\end{array}$ \\
\hline SF2 & 1066 & 10 & 3 & $\begin{array}{l}\text { Number of times common, moderate and severe } \\
\text { solar flares were observed in a } 24 \mathrm{~h} \text { interval. }\end{array}$ \\
\hline Jura & 359 & 15 & 3 & $\begin{array}{l}\text { Concentration of heavy metals in the top soil of a } \\
\text { region in Swiss called Jura. }\end{array}$ \\
\hline WQ & 1060 & 16 & 14 & $\begin{array}{l}\text { Relative representation of plant and animal species } \\
\text { in Slovenian rivers based on physical and chemical } \\
\text { water quality parameters. }\end{array}$ \\
\hline ENB & 768 & 8 & 2 & $\begin{array}{l}\text { Heating and cooling load required for energy } \\
\text { efficient buildings based on their dimensions and } \\
\text { material choice. }\end{array}$ \\
\hline Slump & 103 & 7 & 3 & $\begin{array}{l}\text { Slump, flow and compressive strength concrete } \\
\text { properties based on the amount of different } \\
\text { concrete ingredients. }\end{array}$ \\
\hline Andro & 49 & 30 & 6 & $\begin{array}{l}\text { Water quality attributes in Thermaikos Gulf of } \\
\text { Thessaloniki, Greece. }\end{array}$ \\
\hline OSALES & 639 & 413 & 12 & $\begin{array}{l}\text { Online monthly sales in the first twelve months } \\
\text { after a product launch. }\end{array}$ \\
\hline SCFP & 1137 & 23 & 3 & $\begin{array}{l}\text { Number of views, clicks and comments on online } \\
\text { issues. }\end{array}$ \\
\hline
\end{tabular}




\section{Appendix B: Obtained Condensed Chaining} Tree Graphs and Target Labels

Table 5 Targets' labels for datasets EDM, ENB and Jura.

\begin{tabular}{llll}
\hline Label & EDM & ENB & Jura \\
\hline T1 & DFlow & Y1 & Cd \\
T2 & DGap & Y2 & Co \\
T3 & - & - & $\mathrm{Cu}$ \\
\hline
\end{tabular}

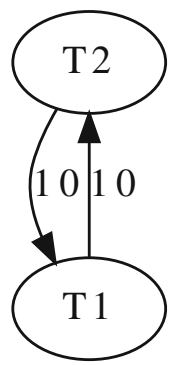

EDM

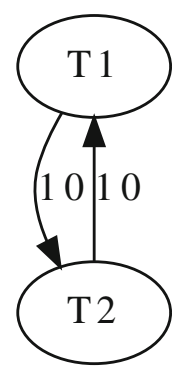

ENB

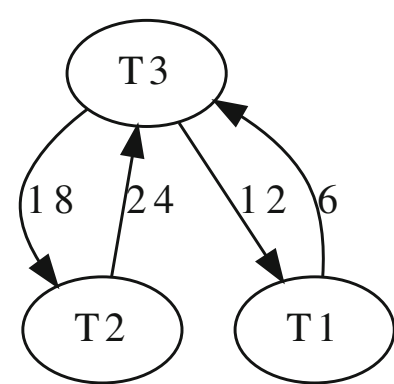

SCPF

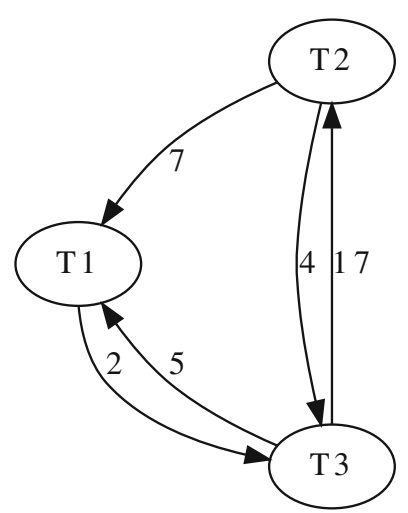

SF2

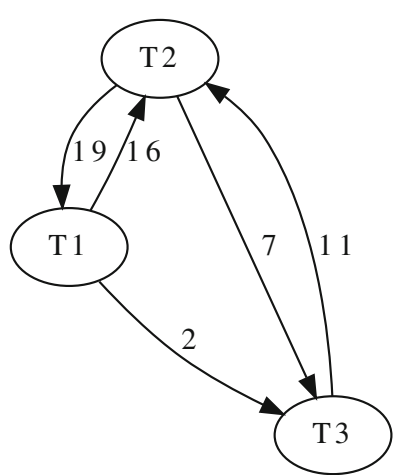

SF1

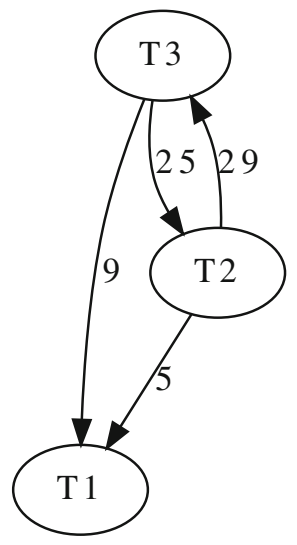

Slump

Figure 5 CCTG for datasets SCPF, SF1, SF2 and Slump.

Figure 4 CCTG for datasets EDM, ENB and Jura.

Table 6 Targets labels for datasets SCPF, SF1, SF2 and Slump.

\begin{tabular}{lllll}
\hline Label & SCPF & SF1 & SF2 & Slump \\
\hline T1 & num_comments & c-class & c-class & Compressive_Strength_Mpa \\
T2 & num_views & m-class & m-class & FLOW_cm \\
T3 & num_votes & x-class & x-class & SLUMP_cm \\
\hline
\end{tabular}


Table 7 Targets' labels for datasets Andro, ATP1D and ATP7D.

Figure 6 CCTG for datasets Andro, ATP1D and ATP7D.

\begin{tabular}{llll}
\hline Label & Andro & ATP1D & ATP7D \\
\hline T1 & Target & LBL+aCOminpA+fut_001 & LBL+aCOminpA+bt7d_000 \\
T2 & Target_2 & LBL+aDLminpA+fut_001 & LBL+aDLminpA+bt7d_000 \\
T3 & Target_3 & LBL+aFLminpA+fut_001 & LBL+aFLminpA+bt7d_000 \\
T4 & Target_4 & LBL+ALLminp0+fut_001 & LBL+ALLminp0+bt7d_000 \\
T5 & Target_5 & LBL+ALLminpA+fut_001 & LBL+ALLminpA+bt7d_000 \\
T6 & Target_6 & LBL+aUAminpA+fut_001 & LBL+aUAminpA+bt7d_000 \\
\hline
\end{tabular}

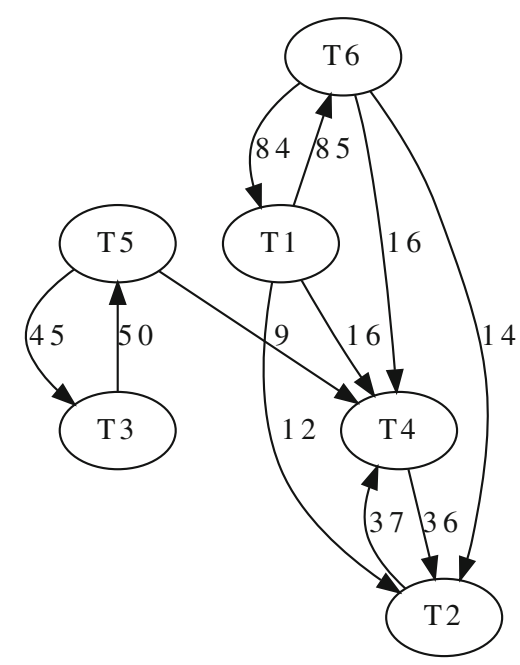

ATP7D
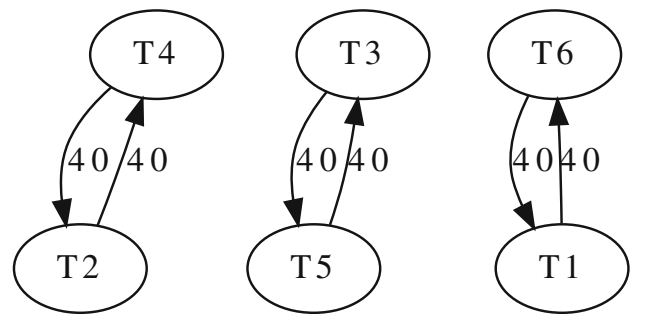

ATP1D 
Table 8 Targets' labels for dataset OES10.

\begin{tabular}{ll}
\hline Label & OES10 \\
\hline T1 & 119032_Education_Administrators_Elementary_and_Secondary_School \\
T2 & 151131_Computer_Programmers \\
T3 & 151141_Database_Administrators \\
T4 & 172141_Mechanical_Engineers \\
T5 & 291051_Pharmacists \\
T6 & 291069_Physicians_and_Surgeons__All_Other \\
T7 & 291127_Speech-Language_Pathologists \\
T8 & 292037_Radiologic_Technologists_and_Technicians* \\
T9 & 292071_Medical_Records_and_Health_Information_Technicians \\
T10 & 392021_Nonfarm_Animal_Caretakers \\
T11 & 412021_Counter_and_Rental_Clerks \\
T12 & 419022_Real_Estate_Sales_Agents \\
T13 & 431011_First-Line_Supervisors_of_Office_and_Administrative_Support_Workers \\
T14 & 432011_Switchboard_Operators_Including_Answering_Service \\
T15 & 513021_Butchers_and_Meat_Cutters \\
T16 & 519061_Inspectors_Testers_Sorters_Samplers_and_Weighers \\
\hline
\end{tabular}

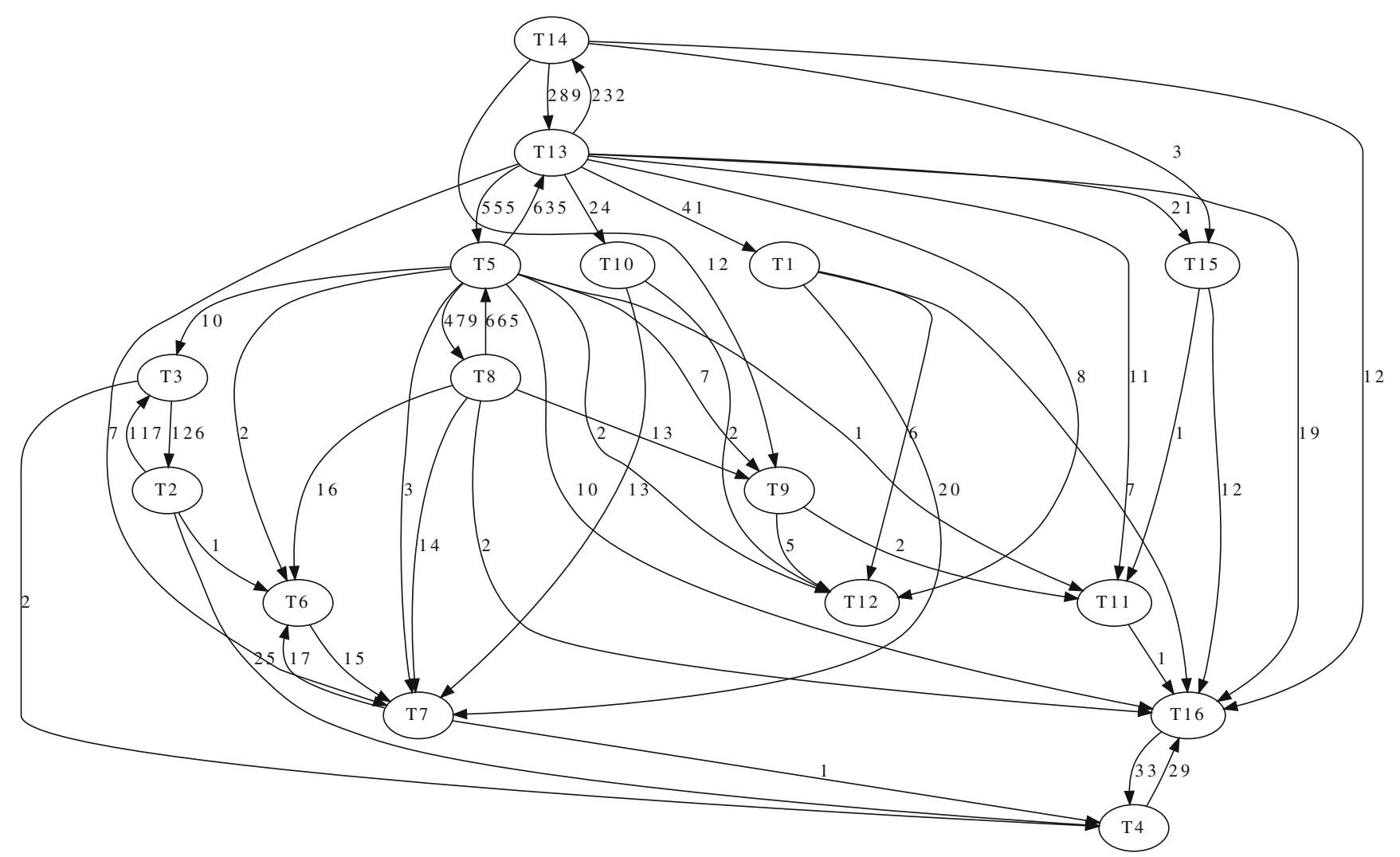

Figure 7 CCTG for dataset OES10. 
Table 9 Targets' labels for dataset OES97.

\begin{tabular}{ll}
\hline Label & OES97 \\
\hline T1 & 13008_Purchasing_Managers \\
T2 & 15014_Industrial_Production_Managers \\
T3 & 15017_Construction_Managers \\
T4 & 21114_Accountants_and_Auditors \\
T5 & 27108_Psychologists \\
T6 & 27311_Recreation_Workers \\
T7 & 32314_Speech-Language_Pathologists_and_Audiologists \\
T8 & 32511_Physician_Assistants \\
T9 & 53905_Teacher_Aides_and_Educational_Assistants_Clerical \\
T10 & 58028_Shipping_Receiving_and_Traffic_Clerks \\
T11 & 65032_Cooks_Fast_Food \\
T12 & 85110_Machinery_Maintenance_Mechanics \\
T13 & 92965_Crushing_Grinding_Mixing_and_Blending_Machine_Operators_and_Tenders \\
T14 & 92998_All_Other_Machine_Operators_and_Tenders \\
T15 & 98502_Machine_Feeders_and_Offbearers \\
T16 & 98902_Hand_Packers_and_Packagers \\
\hline
\end{tabular}

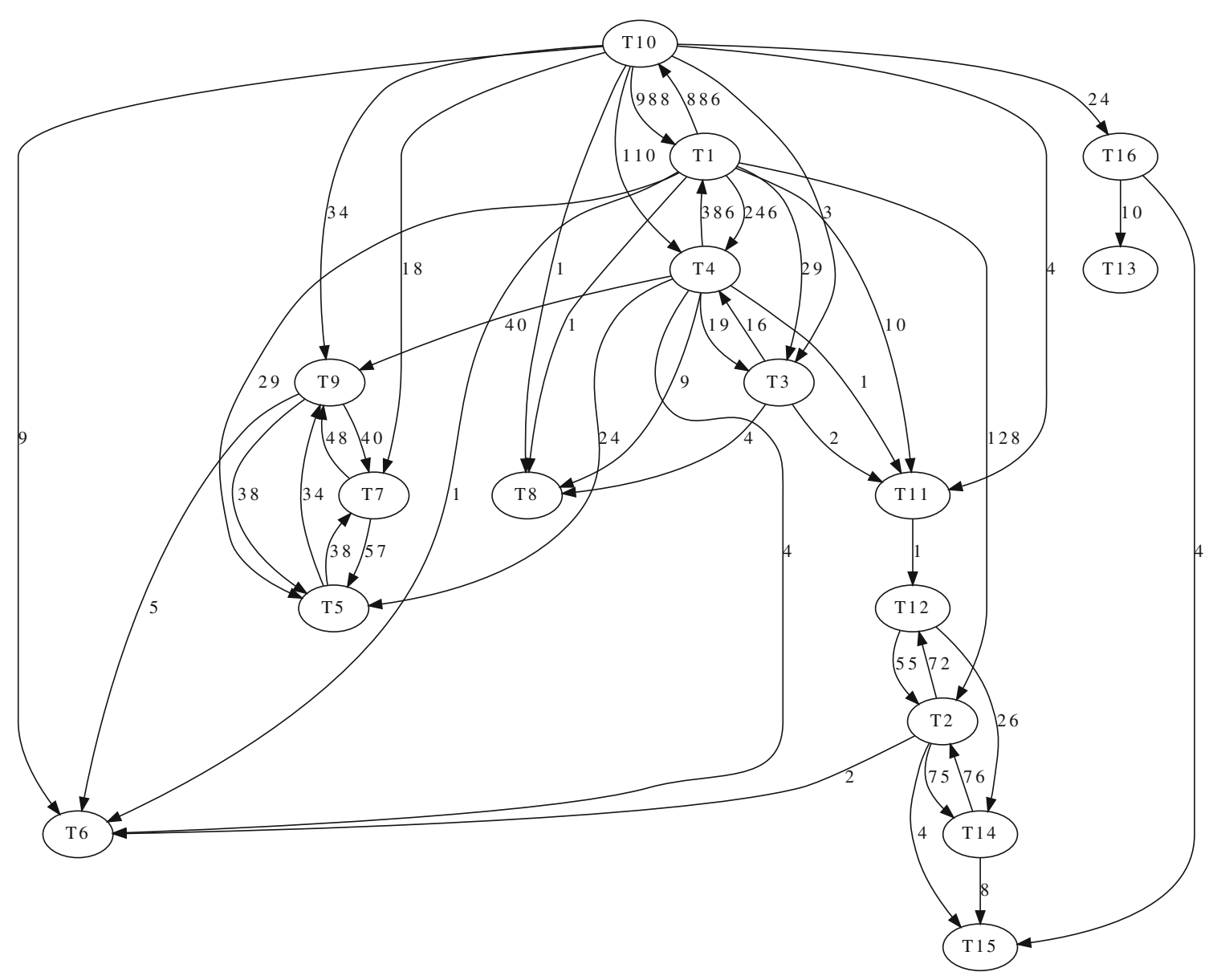

Figure 8 CCTG for dataset OES97. 
Table 10 Targets labels for datasets OSALES, RF1 and RF2.

\begin{tabular}{|c|c|c|c|c|c|c|c|}
\hline Label & OSALES & RF1 & RF2 & Label & SCM1D & SCM20D & WQ \\
\hline $\mathrm{T} 1$ & Outcome_M1 & CHSI2_48H_0 & CHSI2_48H_0 & $\mathrm{T} 1$ & LBL & LBL & 17300 \\
\hline $\mathrm{T} 2$ & Outcome_M2 & CLKM7_48H_0 & CLKM7_48H_0 & $\mathrm{T} 2$ & MTLp2 & MTLp2A & 19400 \\
\hline $\mathrm{T} 3$ & Outcome_M3 & DLDI4_48H_0 & DLDI4_48H_0 & $\mathrm{T} 3$ & MTLp3 & MTLp3A & 25400 \\
\hline $\mathrm{T} 4$ & Outcome_M4 & EADM7_48H_0 & EADM7_48H_0 & $\mathrm{T} 4$ & MTLp4 & MTLp4A & 29600 \\
\hline $\mathrm{T} 5$ & Outcome_M5 & NAPM7_48H_0 & NAPM7_48H_0 & $\mathrm{T} 5$ & MTLp5 & MTLp5A & 30400 \\
\hline T6 & Outcome_M6 & NASI2_48H_0 & NASI2_48H_0 & T6 & MTLp6 & MTLp6A & 33400 \\
\hline $\mathrm{T} 7$ & Outcome_M7 & SCLM7_48H_0 & SCLM7_48H_0 & $\mathrm{T} 7$ & MTLp7 & MTLp7A & 34500 \\
\hline $\mathrm{T} 8$ & Outcome_M8 & VALI2_48H_0 & VALI2_48H_0 & $\mathrm{T} 8$ & MTLp8 & MTLp8A & 37880 \\
\hline T9 & Outcome_M9 & - & - & T9 & MTLp9 & MTLp9A & 38100 \\
\hline $\mathrm{T} 10$ & Outcome_M10 & - & - & $\mathrm{T} 10$ & MTLp10 & MTLp10A & 49700 \\
\hline $\mathrm{T} 11$ & Outcome_M11 & - & - & $\mathrm{T} 11$ & MTLp11 & MTLp11A & 50390 \\
\hline \multirow[t]{5}{*}{$\mathrm{T} 12$} & Outcome_M12 & - & - & $\mathrm{T} 12$ & MTLp12 & MTLp12A & 55800 \\
\hline & & & & $\mathrm{T} 13$ & MTLp13 & MTLp13A & 57500 \\
\hline & & & & $\mathrm{T} 14$ & MTLp14 & MTLp14A & 59300 \\
\hline & & & & $\mathrm{T} 15$ & MTLp15 & MTLp15A & - \\
\hline & & & & $\mathrm{T} 16$ & MTLp16 & MTLp16A & - \\
\hline
\end{tabular}

Table 11 Targets labels for dataset SCM1D, SCM20D and WQ.

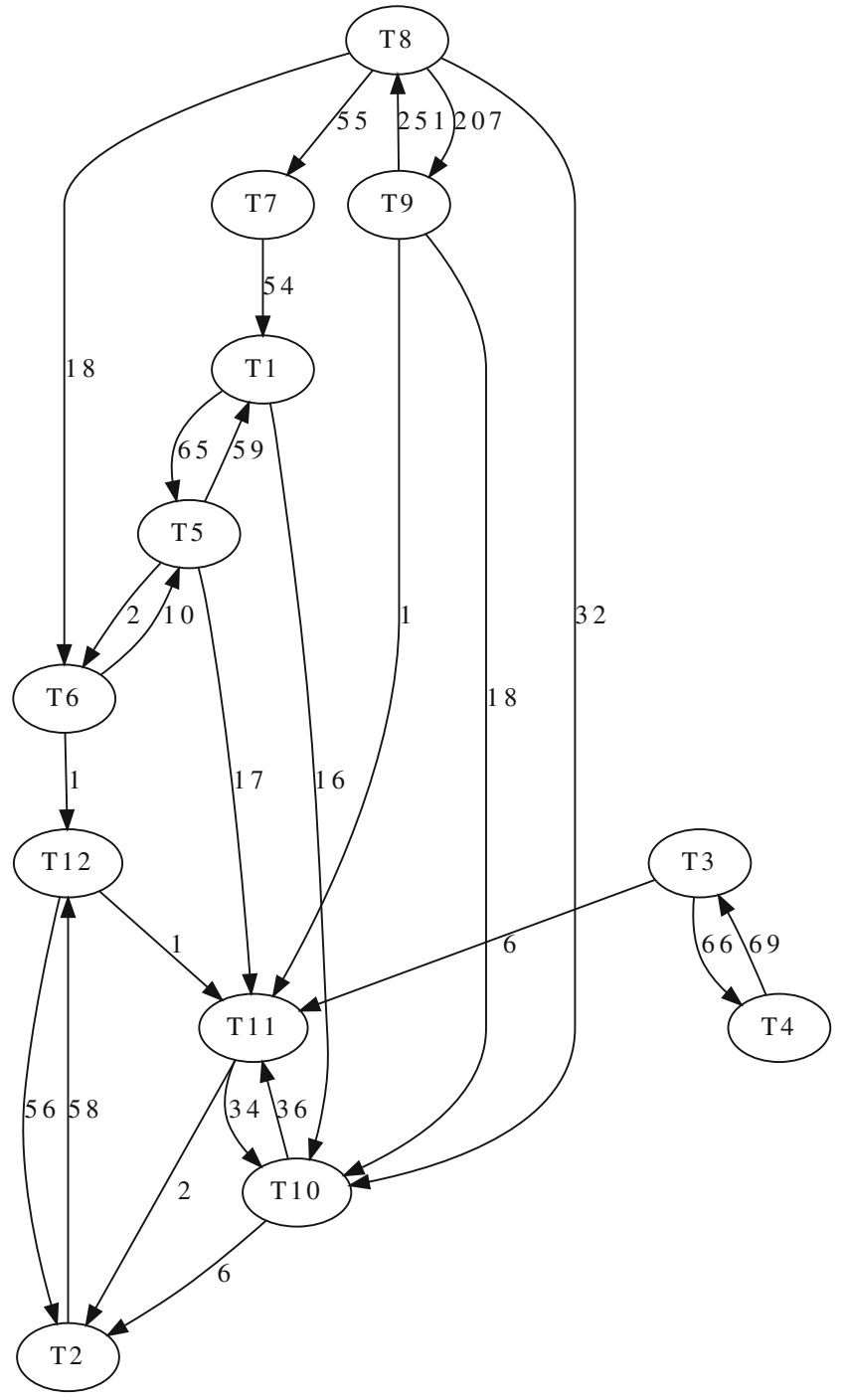

Figure 9 CCTG for dataset OSALES. 
Figure $10 \mathrm{CCTG}$ for datasets RF1 and RF2.
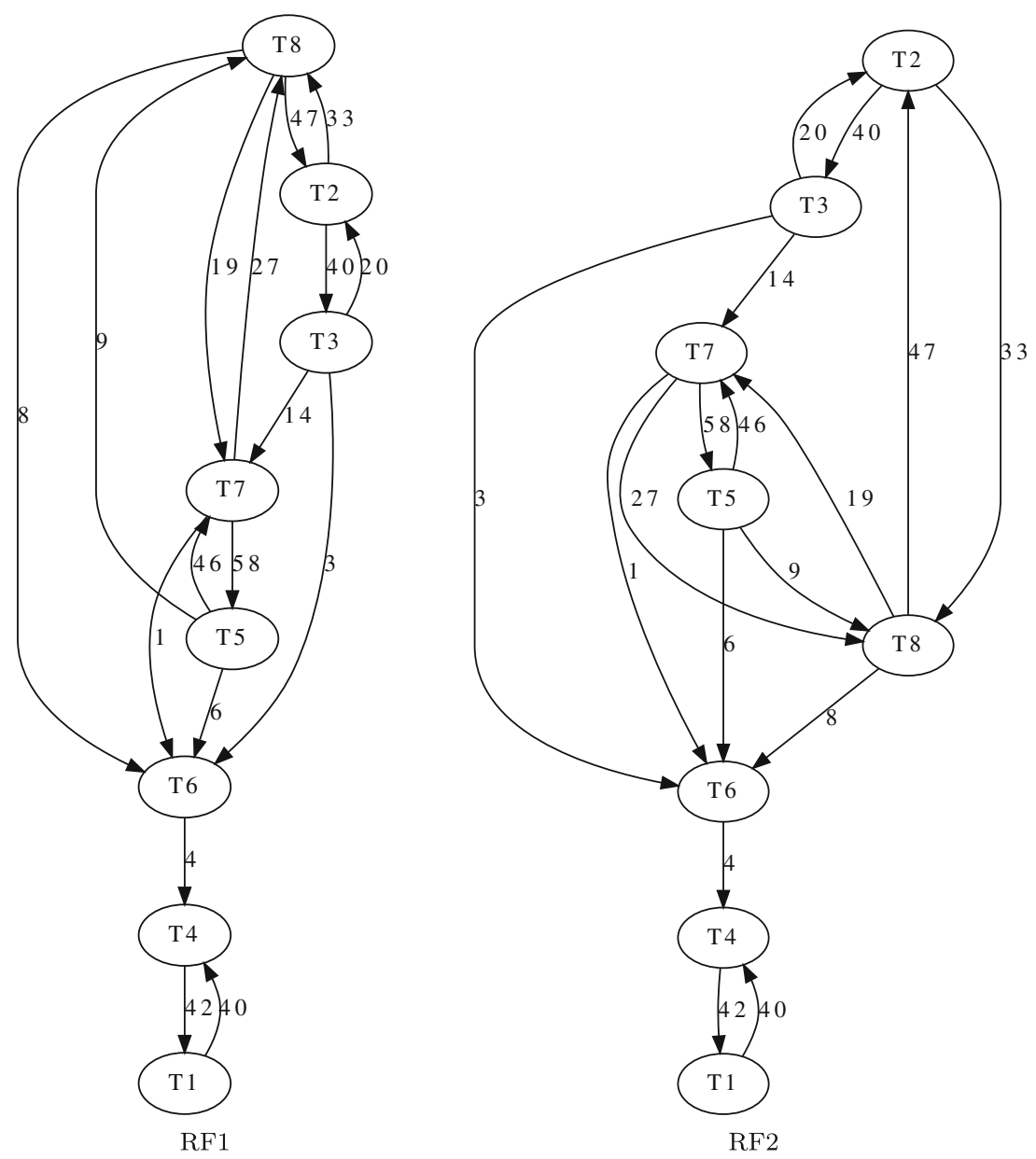
Figure 11 CCTG for datasets SCM1D and SCM20D.
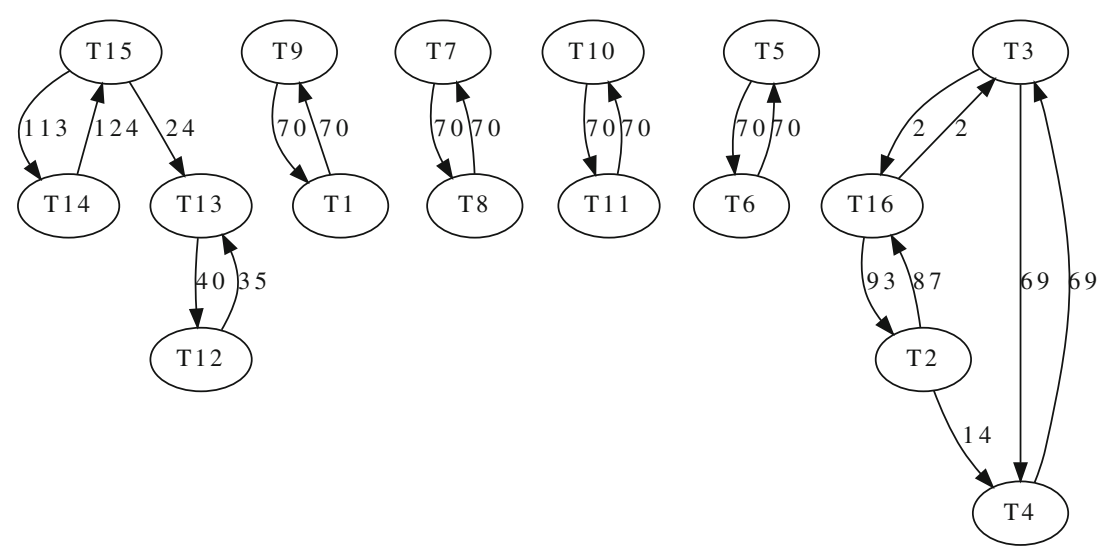

SCM1D

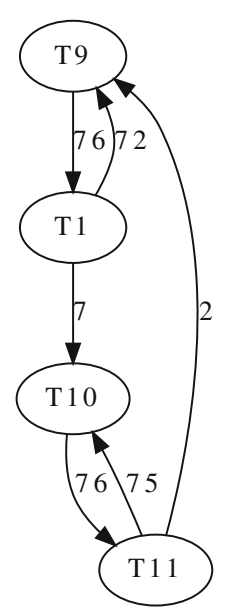

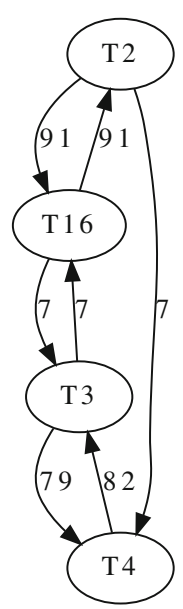

SCM20D

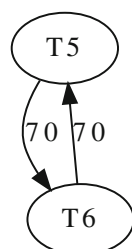

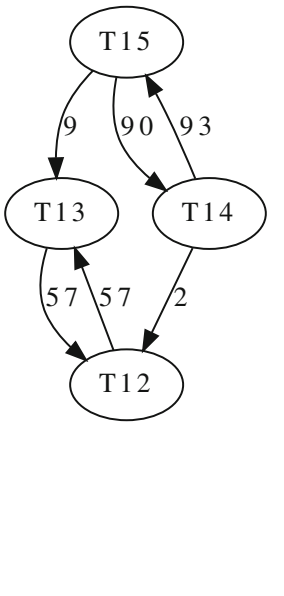




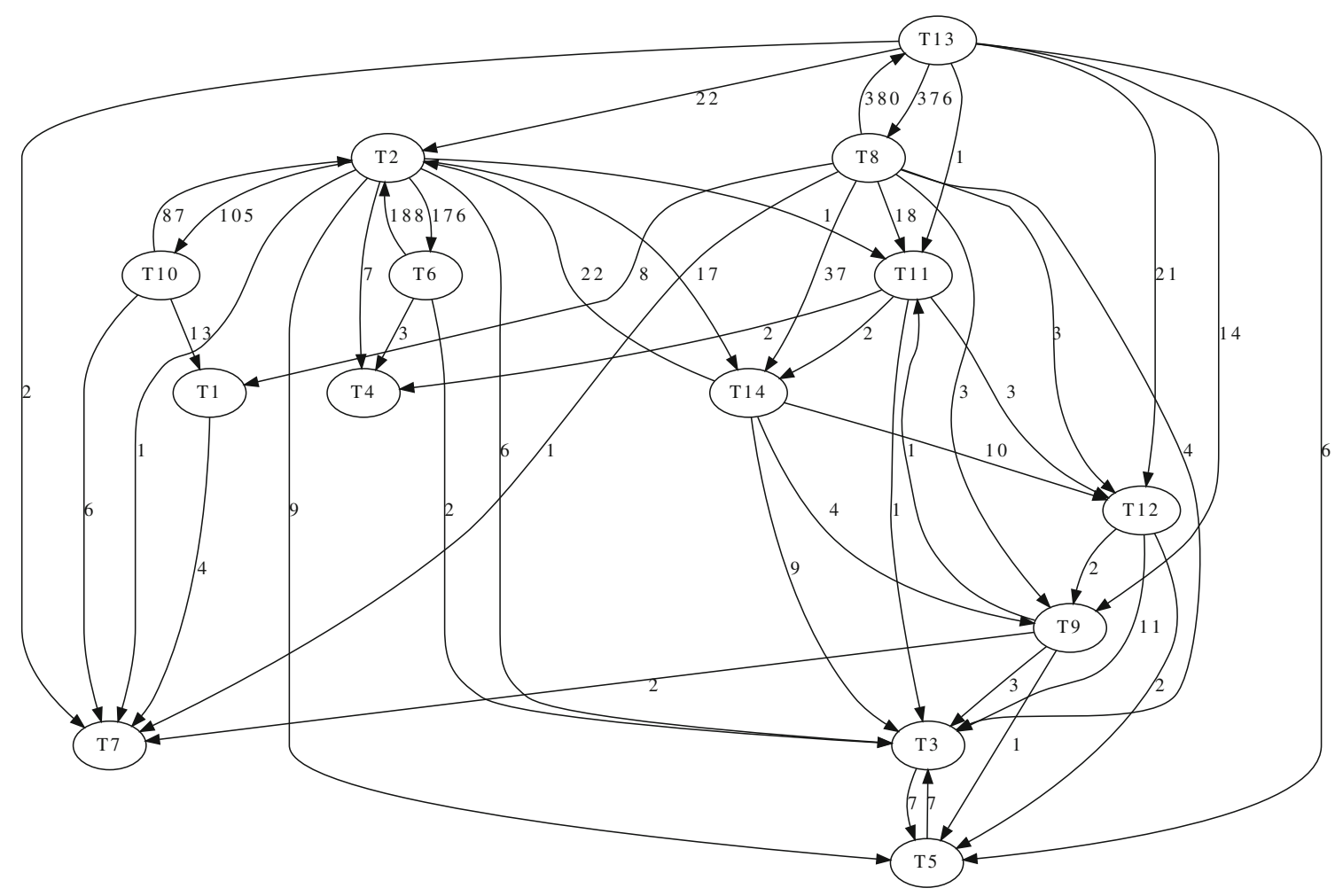

Figure 12 CCTG for dataset WQ.

\section{References}

1. Aho, T., Zenko, B., Dzeroski, S., Elomaa, T. (2012). Multi-target regression with rule ensembles. Journal of Machine Learning Research, 13, 2367-2407.

2. Borchani, H., Varando, G., Bielza, C., Larrañaga, P. (2015). A survey on multi-output regression. Wiley Interdisciplinary Reviews: Data Mining and Knowledge Discovery, 5(5), 216-233.

3. Breiman, L. (2001). Random forests. Machine learning, 45.1, 5-32. https://doi.org/10.1017/CBO9781107415324.004.

4. Brugger, D., Rosenstiel, W., Bogdan, M. (2011). Online SVR training by solving the primal optimization problem. Journal of Signal Processing Systems, 65(3), 391-402.

5. Chen, H., \& Ser, W. (2011). Sound source DOA estimation and localization in noisy reverberant environments using least-squares support vector machines. Journal of Signal Processing Systems, 63(3), 287-300.

6. Cortes, C., \& Vapnik, V. (1995). Support-vector networks. Machine Learning, 20(3), 273-297. https://doi.org/10.1023/A:10 22627411411.

7. Demšar, J. (2006). Statistical comparisons of classifiers over multiple data sets. The Journal of Machine Learning Research, 7, 1-30.

8. Di Persio, L., \& Honchar, O. (2016). Artificial neural networks architectures for stock price prediction: comparisons and applications. International Journal of Circuits, Systems and Signal Processing, 10, 403-413.
9. Drucker, H., Burges, C.J.C., Kaufman, L., Smola, A.J., Vapnik, V. (1997). Support vector regression machines. In Mozer, M.C., Jordan, M.I., Petsche, T. (Eds.) Advances in neural information processing systems (Vol. 9, pp. 155-161). MIT Press. http://papers. nips.cc/paper/1238-support-vector-regression-machines.pdf.

10. Evgeniou, T., Figueiras-Vidal, A.R., Theodoridis, S. (2008). Emerging machine learning techniques in signal processing.

11. Gama, J., \& Brazdil, P. (2000). Cascade generalization. Machine Learning, 41(3), 315-343. https://doi.org/10.1023/A:100765211 114878.

12. Genuer, R., Poggi, J.M., Tuleau-Malot, C. (2010). Variable selection using random forests. Pattern Recognition Letters, 31(14), 2225-2236. https://doi.org/10.1016/j.patrec.2010.03.014. http:// www.sciencedirect.com/science/article/pii/S0167865510000954.

13. Hoeffding, W. (1963). Probability inequalities for sums of bounded random variables. Journal of the American Statistical Association, 58(301), 13-30. https://doi.org/10.1080/01621459. 1963.10500830. http://amstat.tandfonline.com/doi/abs/10.1080/ 01621459.1963.10500830.

14. Katagiri, S., Nakamura, A., Adali, T., Tao, J., Larsen, J., Tan, T. (2014). Guest editorial: Machine learning for signal processing. Journal of Signal Processing Systems, 74(3), 281-283. https://doi.org/10.1007/s11265-014-0871-6.

15. Kocev, D., Džeroski, S., White, M.D., Newell, G.R., Griffioen, P. (2009). Using single- and multi-target regression trees and ensembles to model a compound index of vegetation condition. Ecological Modelling, 220(8), 1159-1168. 
16. Kocev, D., Vens, C., Struyf, J., Džeroski, S. (2007). Ensembles of multi-objective decision trees. In European conference on machine learning (pp. 624-631). Springer.

17. Kocev, D., Vens, C., Struyf, J., Džeroski, S. (2013). Tree ensembles for predicting structured outputs. Pattern Recognition, 46(3), 817-833

18. Li, X., \& Zheng, J. (2016). Active learning for regression with correlation matching and labeling error suppression. IEEE Signal Processing Letters, 23(8), 1081-1085.

19. Lichman, M. (2013). UCI machine learning repository. http:// archive.ics.uci.edu/ml

20. Mastelini, S.M., Santana, E.J., Cerri, R., Barbon, S. Jr. (2017). DSTARS: a multi-target deep structure for tracking asynchronous regressor stack. In Brazilian conference on intelligent systems. BRACIS 2017.

21. Melki, G., Cano, A., Kecman, V., Ventura, S. (2017). Multitarget support vector regression via correlation regressor chains. Information Sciences, 415, 53-69.

22. Moyano, J.M., Gibaja, E.L., Ventura, S. (2017). An evolutionary algorithm for optimizing the target ordering in ensemble of regressor chains. In 2017 IEEE congress on evolutionary computation (CEC) (pp. 2015-2021). IEEE.

23. Santana, E.J., Mastelini, S.M., Barbon, S. Jr. (2017). Deep regressor stacking for air ticket prices prediction. In Brazilian symposium of information systems (pp. 216-233). SBSI 2017.

24. Sidike, P., Krieger, E., Alom, M.Z., Asari, V.K., Taha, T. (2017). A fast single-image super-resolution via directional edge-guided regularized extreme learning regression. In Signal, image and video processing (pp. 1-8).

25. Spyromitros-Xioufis, E., Tsoumakas, G., Groves, W., Vlahavas, I. (2016). Multi-target regression via input space expansion: treating targets as inputs. Machine Learning, 104(1), 55-98.

26. Tsoumakas, G., Spyromitros-Xioufis, E., Vrekou, A., Vlahavas, I. (2014). Multi-target regression via random linear target combinations. In Joint european conference on machine learning and knowledge discovery in databases (pp. 225-240). Springer.

27. Wang, Q., Wu, Y., Shen, Y., Liu, Y., Lei, Y. (2015). Supervised sparse manifold regression for head pose estimation in $3 \mathrm{~d}$ space. Signal Processing, 112, 34-42.

28. Watanabe, S., Nakamura, A., Juang, B.H.F. (2014). Structural bayesian linear regression for hidden Markov models. Journal of Signal Processing Systems, 74(3), 341-358.

29. Zhang, W., Liu, X., Ding, Y., Shi, D. (2012). Multi-output LS-SVR machine in extended feature space. In CIMSA 2012 - 2012 IEEE Int. Conf. Comput. Int.ll. Meas. Syst. Appl. Proc. (pp. 130-144). https://doi.org/10.1109/CIMSA.2012.6269600.

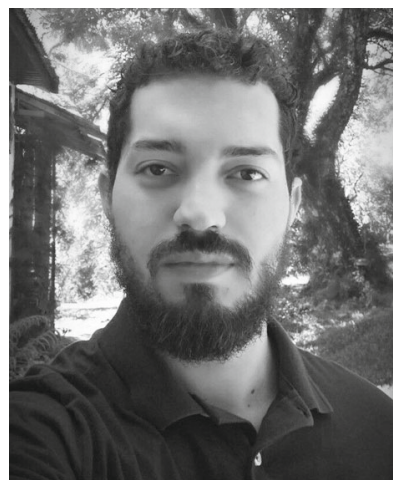

Saulo Martiello Mastelini received his BSc and MSc degrees in Computer Science in 2016 and 2018, respectively, at the State University of Londrina, Brazil. During his bachelor he researched in Computer Graphics, Computer Vision and Numerical methods for solving Partial Differential Equations. During his Master's he focused in Machine Learning techniques, with special attention for Multi-target regression tasks.

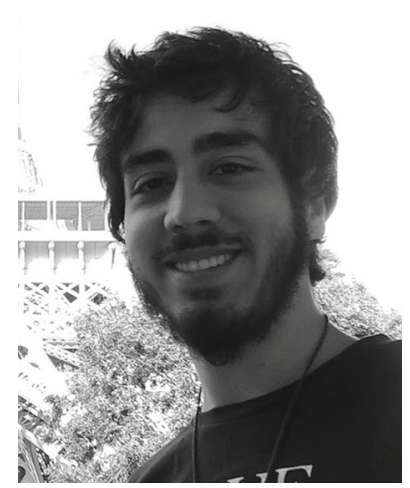

Victor Guilherme Turrisi da Costa BSc, is an MSc student in the Computer Science Department at State University of Londrina (UEL), Brazil. He received his $\mathrm{BSc}$ degree in Computer Science in 2017 at the State University of Londrina (UEL), Brazil. His research interests include Pattern Recognition, Machine Learning and botnet detection.

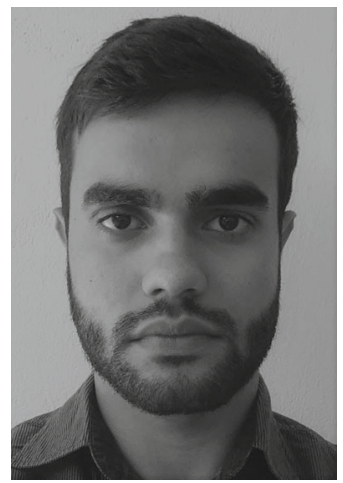

Everton Jose Santana is a Bsc student of Electrical Engineering at State University of Londrina, Brazil. From 2015 to 2016, he was an exchange student at Hanze University of Applied Sciences, the Netherlands, where he followed minors in Biomedical and Sensor System Engineering. His main research topics are Applied Mathematics, Instrumentation/Biomedical Engineering and Machine Learning.

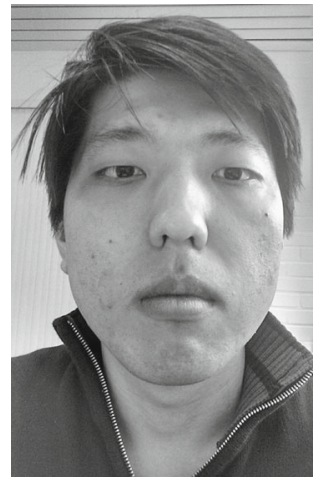

Felipe Kenji Nakano received his BSc degree in Computer Science in 2016 at the State University of Londrina, Brazil. During his bachelor he worked with software development and researched Process Mining. Currently, he is finishing his MSc in Computer Science at Federal University of Sao Carlos. His topics of interest are Hierarchical Classification, Deep Learning and Active Learning. 


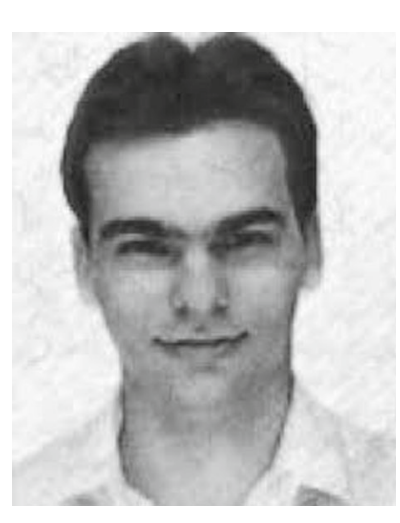

\section{Dr. Rodrigo Capobianco} Guido received his BSc degrees in Computer Science and in Computer Engineering, his MSc degree in Electrical Engineering and his $\mathrm{PhD}$ degree in Computational Applied Physics, respectively from São Paulo State University (UNESP) at São José do Rio Preto - Brazil in 1998, from Educational Foundation at Votuporanga (FEV) - Brazil in 2003, from Campinas State University (UNICAMP) Brazil in 2000 and from University of São Paulo (USP) at São Carlos - Brazil in 2003. From both $\mathrm{BScs}$ to $\mathrm{PhD}$, all his titles focused on signal processing. In complement, he has already participated in two post-doctoral programs in signal processing at USP, from 2003 to 2007, and obtained the title of Associate Professor (Livre-Docência) in signal processing from the School of Engineering at São Carlos - Brazil (USP) in 2008. Dr. Guido has taught signal processing and electronics since 1999 and has published hundreds of scientific articles in IEEE and Elsevier journals, magazines, and conference proceedings. He is serving, or recently served, as an area-editor, as an associate-editor, and as a guest-editor for respected scientific journals, such as IEEE Signal Processing Magazine, IEEE Transactions on Audio, Speech and Language Processing, Elsevier Pattern Recognition Letters, Elsevier Neurocomputing, Elsevier Computers in Biology and Medicine, just to mention a few. Complementarily, he has served as an organiser and chairman for many IEEE conferences along the years. Dr. Guido has received several grants and awards from Brazilian agencies, especially from The State of São Paulo Research Foundation (FAPESP) and from National Council of Research and Development (CNpQ). He has also supervised many theses in his field and is a senior member of the IEEE. Currently, he is an associate professor at UNESP in São José do Rio Preto - Brazil. Dr. Guido has focused his research activities on digital signal processing (DSP) with particular focus on speech processing, i.e., speech transmission and reception, speech enhancement, speech recognition, speaker identification and verification, speech analysis for biomedical purposes, voice morphing, speech emotion classification, voice activity detection, speech synthesis, and so on, specially based on wavelets associated with algorithms for machine learning.

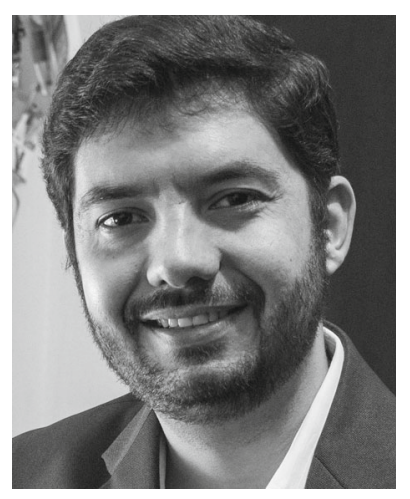

Ricardo Cerri obtained his Bachelor in Computer Science from São Paulo State University (UNESP/Brazil), and his $\mathrm{MSc}$ and $\mathrm{PhD}$ in Computer Science and Computational Mathematics from University of São Paulo (ICMC/USP/Brazil). He has experience working mainly with Bioinformatics and Machine Learning, focusing on advanced methods for data classification, such as multi-output and structured learning. Currently he holds the position of Assistant Professor at the Department of Computer Science from Federal University of São Carlos (UFSCar/Brazil).

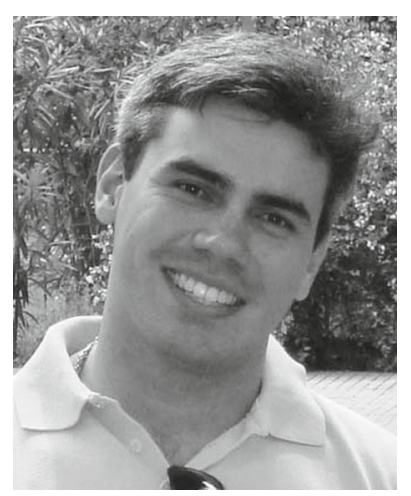

Sylvio Barbon Jr. $\mathrm{PhD}$, is Associate Professor and leader of the research group that studies machine learning in the Computer Science Department at State University of Londrina (UEL), Brazil. He received his $\mathrm{BSc}$ degree in Computer Science in 2005, MSc degree in Computational Physics from University of São Paulo (2007), degree in Computational Engineering during 2008 and $\mathrm{PhD}$ degree (2011) from IFSC/USP such as the MSc degree. During 2017, he was visiting researcher at University of Modena and Reggio Emilia (Italy) working on multispectral analysis and machine learning. Still in 2017, as visiting researcher at Universita Degli Studi Di Milano (Italy) focused on Stream and Process Mining. He is currently a professor in postgraduate and graduate programs. His research interests include Digital Signal Processing, Pattern Recognition and Machine Learning. 